\title{
Change-Of-Bases Abstractions For Non-Linear Hybrid Systems.
}

\author{
Sriram Sankaranarayanan \\ Department of Computer Science, \\ University of Colorado, Boulder, CO, USA. \\ srirams@ colorado.edu
}

\begin{abstract}
We present abstraction techniques that transform a given non-linear dynamical system into a linear system, or more generally, an algebraic system described by polynomials of bounded degree, so that invariant properties of the resulting abstraction can be used to infer invariants for the original system. The abstraction techniques rely on a change-of-bases transformation that associates each state variable of the abstract system with a function involving the state variables of the original system. We present conditions under which a given change-of-bases transformation for a non-linear system can define an abstraction. Furthermore, the techniques developed here apply to continuous systems defined by Ordinary Differential Equations (ODEs), discrete systems defined by transition systems and hybrid systems that combine continuous as well as discrete subsystems.

The techniques presented here allow us to discover, given a non-linear system, if a change-of-bases transformation involving degree-bounded polynomials yielding an algebraic abstraction exists. If so, our technique yields the resulting abstract system, as well. Our techniques enable the use of analysis techniques for linear systems to infer invariants for non-linear systems. We present preliminary evidence of the practical feasibility of our ideas using a prototype implementation.
\end{abstract}

Keywords: Hybrid Systems, Abstraction, Verification, Linearization, Invariants, Conservation Laws, Program Analysis, Abstract Interpretation.

\section{Introduction}

In this paper, we explore a class of abstractions for non-linear autonomous systems (continuous, discrete and hybrid systems) using Change-of-Bases ( $\mathrm{CoB})$ transformations. CoB transformations are obtained for a given system by expressing the dynamics of the system in terms of a new set of variables that relate to the original system variables by means of a transformation. Such a transformation is akin to studying the system under a new set of "bases". We derive conditions on the transformations such that (a) the CoB transformations also define an autonomous system and (b) the resulting system abstracts the

Preprint submitted to Nonlinear Analysis: Hybrid systems

May 5, 2015

(C) 2015. This manuscript version is made available under the Elsevier user license http://www.elsevier.com/open-access/userlicense/1.0/ 
original system: i.e., all invariants of the abstract system can be inverted to obtain invariants for the original system. Furthermore, we often seek abstract systems through $\mathrm{CoB}$ transformations whose dynamics are of a simpler form, more amenable to automatic verification techniques. For instance, it is possible to use $\mathrm{CoB}$ transformations that relate an ODE with non-linear right-hand sides to an affine ODE, or transformations that reduce the degree of a system with polynomial right-hand sides. If such transformations can be found, then safety analysis techniques over the simpler abstract system can be used to infer safety properties of the original system.

In this paper, we make two main contributions: (a) we define CoB transformations for continuous, discrete and hybrid systems and provide conditions under which a given transformation is valid; (b) we provide search techniques for finding $\mathrm{CoB}$ transformations that result in a polynomial system whose righthand sides are degree limited by some limit $d \geq 1$. Specifically, the case $d=1$ yields an affine abstraction; and (c) we provide experimental evidence of the application of our techniques to a variety of ordinary differential equations (ODEs) and discrete programs.

The results in this paper extend our previously published results that appeared in Hybrid Systems: Computation and Control (HSCC) 2011 [52]. The contributions of this paper include (a) an extension from linearizing $\mathrm{CoB}$ transformations to degree-bounded polynomial $\mathrm{CoB}$ transformations, (b) extending the theory from purely continuous system to purely hybrid systems, and (c) an improved implementation that can handle hybrid systems with some evaluation results over this implementation. On the other hand, our previous work also included an extension of the theory to differential inequalities and iterative techniques over cones. These extensions are omitted here. Finally, our work here is restricted to autonomous ODEs and programs without external inputs. All uncertainty in our system is restricted to the initial set of states, which are not considered explicitly in the process of abstracting the dynamics, as detailed in Section 2.2. Bounded disturbances can potentially be handled in a planned extension of this paper that considers iterations over cones rather than vector spaces [52].

\subsection{Motivating Examples}

In this section, we motivate the techniques developed in this paper by means of a few illustrative examples involving purely continuous ODEs and purely discrete programs.

Our first example concerns a continuous system defined by a system of Ordinary Differential Equations (ODEs):

Example 1.1. Consider a continuous system over $\{x, y\}: \dot{x}=x y+2 x, \quad \dot{y}=$ $-\frac{1}{2} y^{2}+7 y+1$, with initial conditions given by the set $x \in[0,1], y \in[0,1]$. Using the transformation $\alpha:(x, y) \mapsto\left(w_{1}, w_{2}, w_{3}\right)$ wherein $\alpha_{1}(x, y)=x, \alpha_{2}(x, y)=x y$ and $\alpha_{3}(x, y)=x y^{2}$, we find that the dynamics over $\vec{w}$ can be written as

$$
\dot{w}_{1}=2 w_{1}+w_{2}, \quad \dot{w}_{2}=w_{1}+9 w_{2}+\frac{1}{2} w_{3}, \dot{w_{3}}=2 w_{2}+16 w_{3} .
$$


Its initial conditions are given by $w_{1} \in[0,1], w_{2} \in[0,1], w_{3} \in[0,1]$. We analyze the system using the TimePass tool as presented in our previous work [56] to obtain polyhedral invariants:

$$
\begin{aligned}
& -w_{1}+2 w_{2} \geq-1 \wedge w_{3} \geq 0 \wedge w_{2} \geq 0 \wedge \\
& -16 w_{1}+32 w_{2}-w_{3} \geq-17 \wedge 32 w_{2}-w_{3} \geq-1 \wedge \\
& 2 w_{1}-4 w_{2}+17 w_{3} \geq-4 \wedge 286 w_{1}-32 w_{2}+w_{3} \geq-32 \wedge \\
& \cdots
\end{aligned}
$$

Substituting back, we can infer polynomial inequality invariants on the original system including,

$$
\begin{aligned}
& -x+2 x y \geq-1 \wedge x y^{2} \geq 0 \wedge-16 x+32 x y-x y^{2} \geq-17 \\
& x \geq 0 \wedge 2 x-4 x y+17 x y^{2} \geq-4 \wedge \cdots
\end{aligned}
$$

Finally, we link the presence of affine change-of-bases abstractions with a set of conserved quantities (first integrals) of the original system (see Lemma 2.4 in page 21). Recall that a conserved quantity $f(\vec{x}, t)$ is a function whose value is constant over all trajectories: i.e, for all time trajectories of the system $f(\vec{x}(t), t)=f(\vec{x}(0), 0)$. For this example, we infer the following conserved quantity on the underlying non-linear system:

$$
\begin{aligned}
& \left(\frac{e^{-9 t}}{51}+\frac{1}{102}(50+7 \sqrt{51}) e^{(-9+\sqrt{51}) t}+\frac{1}{102}(50-7 \sqrt{51}) e^{-(9+\sqrt{51}) t}\right) x+ \\
& \left(-\frac{1}{102} e^{-9 t-(9+\sqrt{51}) t}\left(\begin{array}{l}
7 e^{9 t}-\sqrt{51} e^{9 t}-14 e^{(9+\sqrt{51}) t}+ \\
7 e^{9 t+(-9+\sqrt{51}) t+(9+\sqrt{51}) t}+ \\
\sqrt{51} e^{9 t+(-9+\sqrt{51}) t+(9+\sqrt{51}) t}
\end{array}\right)\right) x y+ \\
& \left(\frac{1}{204} e^{-9 t-(9+\sqrt{51}) t}\left(e^{9 t}-2 e^{(9+\sqrt{51}) t}+e^{9 t+(-9+\sqrt{51}) t+(9+\sqrt{51}) t}\right)\right) x y^{2}
\end{aligned}
$$

Finally, if $x(0) \neq 0$, the map $\alpha$ is invertible and therefore, the ODE above can be integrated.

Note that not every transformation yields a linear abstraction. In fact, most transformations will not define an abstraction. The conditions for an abstraction are discussed in Section 2.

Next, we motivate our approach on purely discrete programs, showing how $\mathrm{CoB}$ transformations can linearize a discrete program with non-linear assignments, modeled by a transition system [33]. In turn, we show how invariants of the abstract linearized program can be transferred back.

Example 1.2. Figure 1 shows an example proposed originally by Petter [43] that considers a program that sums up all squares from 1 to $K^{2}$ for some input $K \geq 0$. Consider a very simple change-of-bases transformation wherein we add a new variable "y2" that tracks the value of $y^{2}$ as the loop is executed. It is straightforward to write assignments for " $\mathrm{y} 2$ " in terms of itself, $x, y$. Finally, the resulting program has affine guards and assignments, making it suitable for polyhedral abstract interpretation $[15,26]$. The polyhedral analysis yields 
Previous work on invariant generation for hybrid system by the author constructs invariants by assuming a desired template form (ansatz) with unknown parameters and applying the "consecution" conditions such as strong consecution and constant scale consecution [55]. Matringe et al. present generalizations of these conditions using morphisms [34]. Therein, they observe that strong and constant scale consecution conditions correspond to a linear abstraction of the original non-linear system of a restrictive form. Specifically, the original system is abstracted by a system of the form $\frac{d x}{d t}=0$ for strong consecution, and a system of the form $\frac{d x}{d t}=\lambda x$ for constant-scale consecution. This paper builds upon this observation by Matringe et al. using fixed-point computation techniques to search for a general linear abstraction that is related to the original system by a change-of-bases transformation. Our work is closely related to the technique of differential invariants proposed by Platzer et al $[44,46]$. A key primitive used in this technique can be cast as a search for abstractions of the form $\frac{d z}{d t}=0$, wherein $z$ stands for an expression involving the state variables of the system of interest. The basic differential invariant framework is extended to allow a larger set of rules involving so-called "cuts" in a subsequent extension [45]. It is shown that the power of differential invariants can be strictly improved by the addition of so-called auxilliary state variables. In contrast, here we attempt a different type of extension by using change-of-bases transformations, so that properties of the "abstract" system resulting from the change-of-bases can indeed be transferred back to the original system. In this regard, our approach allows us to use a rich set of known facts about the trajectories of linear systems that can be transferred back to reason about the original system.

Example 1.3. Consider, once again, our motivating example (Ex. 1.1). We wish to show that the set $x \geq 0 \wedge y \geq 0$ is a positive invariant for the system. However, neither $x \geq 0$ nor $y \geq 0$ can be established as a differential invariant. However, consider the linear system abstraction recalled below:

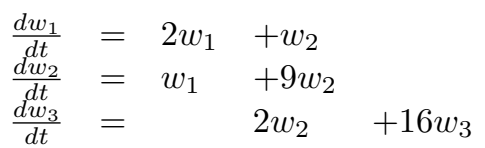

The system has the form $\frac{d \vec{w}}{d t}=A \vec{w}$ where $A$ is a so-called Metzler matrix, i.e, a matrix whose nondiagonal entries are nonnegative [19]. It can be shown that $e^{A t}$ is a positive matrix for each $t \geq 0$. In turn, it follows that the first quadrant $w_{1} \geq 0 \wedge w_{2} \geq 0 \wedge w_{3} \geq 0$ is positive invariant. This immediately shows that in the original co-ordinate system $x \geq 0 \wedge y \geq 0$ is a positive invariant.

Differential invariants are used inside a sophisticated verification framework for hybrid programs called Keymaera ${ }^{1}$. On one hand, work here extends to more general abstractions that can involve multiple variables and can be system specific. However, the integration inside a framework such as Keymaera is an

\footnotetext{
${ }^{1}$ Cf. http://symbolaris.com/info/KeYmaera.html
} 
important problem that is not addressed here. The approach presented here can be generalized to fixed-point computation over cones to search for generalized linear differential inequality abstractions.

Fixed point techniques for deriving invariants of differential equations have been proposed by the author in previous papers $[56,51]$ These techniques have addressed the derivation of polyhedral invariants for affine systems [56] and algebraic invariants for systems with polynomial right-hand sides [51]. In this technique, we employ the machinery of fixed-points. Our primary goal is not to derive invariants, per se, but to search for abstractions of non-linear systems into linear systems.

There has been a large body of work focused on the use of algebraic techniques for deriving invariants of programs. Previous work by the author focuses on deriving polynomial equality invariants for programs, automatically, by setting up template polynomial invariants with unknown coefficients and deriving constraints on values of these coefficients to ensure invariance [55, 54]. Carbonell et al. present loop invariant generation techniques by solving recurrences and computing polynomial ideals to capture algebraic properties of the reachable states [50] and subsequently using the descending abstract interpretation over ideals with widening over ideals to ensure termination [49]. The approach is extended to polyhedral cones generated by polynomial inequalities to generate polynomial inequality invariants [4]. Another set of related techniques concern the use of linear invariant generation techniques for polynomial equality invariant generation. Müller-Olm and Seidl explore the use of linear algebraic techniques, wherein a vector space of matrices are used to summarize the transformation from the initial state of a program to a given location. This space is then used to generate polynomial invariants of the program [38]. Likewise, the work of Colón explores degree-bounded restrictions to Nullstellensatz to enable linear algebraic techniques to generate polynomial invariants [14]. More recently, the work of Kovacs uses sophisticated techniques for solving recurrence equations over so-called P-Solvable loops to generate polynomial invariants for them [29].

Finally, our approach is closely related to Carlemann embedding that can be used to linearize a given differential equation with polynomial right-hand sides [30]. The standard Carlemann embedding technique creates an infinite dimensional linear system, wherein, each dimension corresponds to a monomial or a basis polynomial. In practice, it is possible to create a linear approximation with known error bounds by truncating the monomial terms beyond a degree cutoff. Our approach for differential equation abstractions can be seen as a search for a "finite submatrix" inside the infinite matrix created by the Carleman linearization. The rows and columns of this submatrix correspond to monomials such that the derivative of each monomial in the submatrix is a linear combination of monomials that belong the submatrix.

Organization: The rest of this paper presents our approach for Ordinary Differential Equations in Section 2. The ideas for discrete systems are presented in Section 3 by first presenting the theory for simple loops and then extending it to 
arbitrary discrete programs modeled by transition systems. The extensions to hybrid systems are presented briefly by suitably merging the techniques for discrete programs with those for ODEs. Finally, Section 4 presents an evaluation of the ideas presented using our implementation that combines an automatic search for $\mathrm{CoB}$ transformations with polyhedral invariant generation for continuous, discrete and hybrid systems [15, 26, 56].

\section{Abstractions for ODEs}

We first present some preliminary definitions for continuous systems defined by Ordinary Differential Equations (ODEs).

\subsection{Preliminaries: Continuous Systems}

Let $\mathbb{R}$ denote the field of real numbers. Let $x_{1}, \ldots, x_{n}$ denote a set of variables, collectively represented as $\vec{x}$. The set $\mathbb{R}[\vec{x}]$ denotes the ring of multivariate polynomials over $\mathbb{R}$.

A power-product over $\vec{x}$ is of the form $x_{1}^{r_{1}} x_{2}^{r_{2}} \cdots x_{n}^{r_{n}}$, succinctly written as $\vec{x}^{\vec{r}}$, wherein each $r_{i} \in \mathbb{N}$. The degree of a monomial $\vec{x}^{\vec{r}}$ is given by $\sum_{i=1}^{n} r_{i}=\overrightarrow{1} \cdot \vec{r}$. A monomial is of the form $c \cdot m$ where $c \in \mathbb{R}$ and $m$ is a power-product. A multivariate polynomial $p$ is a sum of finitely many monomial terms: $p=$ $\sum_{\vec{r} \in \mathbb{R}^{n}} c_{r} \vec{x}^{\vec{r}}$. The degree of a multivariate polynomial $p$ is the maximum over the degrees of all monomial terms $m$ that occur in $p$ with a non-zero coefficient.

We assume some basic familiarity with the basics of computational algebraic geometry [16] and elementary linear algebra [27].

Vector Fields: A vector field $F$ over a manifold $M \subseteq \mathbb{R}^{n}$ is a map $F: M \mapsto \mathbb{R}^{n}$ from each $\vec{x} \in M$ to a vector $F(\vec{x}) \in \mathbb{R}^{n}$, wherein $F(\vec{x}) \in T_{M}(\vec{x})$, the tangent space of $M$ at $\vec{x}$.

A vector field $F$ is continuous if the map $F$ is continuous. A polynomial vector field $F \in(\mathbb{R}[\vec{x}])^{n}$ is specified by a tuple $F(\vec{x})=\left\langle p_{1}(\vec{x}), p_{2}(\vec{x}), \ldots, p_{n}(\vec{x})\right\rangle$, wherein $p_{1}, \ldots, p_{n} \in \mathbb{R}[\vec{x}]$.

A system of (coupled) ordinary differential equations (ODE) specifies the evolution of variables $\vec{x}:\left(x_{1}, \ldots, x_{n}\right) \in M$ over time $t$ :

$$
\frac{d x_{1}}{d t}=p_{1}\left(x_{1}, \ldots, x_{n}\right), \cdots, \frac{d x_{n}}{d t}=p_{n}\left(x_{1}, \ldots, x_{n}\right),
$$

The system implicitly defines a vector field $F(\vec{x}):\left\langle p_{1}(\vec{x}), \ldots, p_{n}(\vec{x})\right\rangle$. We assume that all vector fields $F$ considered in this paper are (locally) Lipschitz continuous over the domain $M$. In general, all polynomial vector fields are locally Lipschitz continuous, but not necessarily globally Lipschitz continuous over an unbounded domain $X$. The Lipschitz continuity of the vector field $F$, ensures that given $\vec{x}=\vec{x}_{0}$, there exists a time $T>0$ and a unique time trajectory $\tau:[0, T) \mapsto \mathbb{R}^{n}$ such that $\frac{d \tau(t)}{d t}=F(\tau(t))$ for all $t \in[0, T)[35]$. 
Definition 2.1. For a vector field $F:\left\langle f_{1}, \ldots, f_{m}\right\rangle$, the Lie derivative of a smooth function $f(\vec{x})$ is given by

$$
\mathcal{L}_{F}(f)=(\nabla f) \cdot F(\vec{x})=\sum_{i=1}^{n}\left(\frac{\partial f}{\partial x_{i}} \cdot f_{i}\right)
$$

Definition 2.2. A continuous system over variables $x_{1}, \ldots, x_{n}$ consists of a tuple $\mathcal{S}:\left\langle X_{0}, \mathcal{F}, X_{I}\right\rangle$ wherein $X_{0} \subseteq \mathbb{R}^{n}$ is the set of initial states, $\mathcal{F}$ is a vector field over the domain represented by a manifold $X_{I} \subseteq \mathbb{R}^{n}$.

Note that in the context of hybrid systems, the set $X_{I}$ is often referred to as the state invariant or the domain manifold.

\subsection{Change-of-Bases for Continuous Systems}

In this section, we will present change-of-bases ( $\mathrm{CoB})$ transformations of continuous systems and some of their properties.

Consider a map $\alpha: \mathbb{R}^{k} \mapsto \mathbb{R}^{l}$. Given a set $S \subseteq \mathbb{R}^{k}$, let $\alpha(S)$ denote the set obtained by applying $\alpha$ to all the elements of $S$. Likewise, the inverse map over sets is $\alpha^{-1}(T):\{s \mid \alpha(s) \in T\}$. Let $\mathcal{S}:\left\langle X_{0}, \mathcal{F}, X_{I}\right\rangle$ be a continuous system over variables $\vec{x}:\left(x_{1}, \ldots, x_{n}\right)$, involving the vector field $\mathcal{F}$, and $\mathcal{T}:\left\langle Y_{0}, \mathcal{G}, Y_{I}\right\rangle$ be a continuous system over variables $\vec{y}:\left(y_{1}, \ldots, y_{m}\right)$ involving the vector field $\mathcal{G}$.

Definition 2.3. We say that $\mathcal{T}$ simulates $\mathcal{S}$ iff there exists a smooth mapping $\alpha: \mathbb{R}^{n} \mapsto \mathbb{R}^{m}$ such that

1. $Y_{0} \supseteq \alpha\left(X_{0}\right)$ and $Y_{I} \supseteq \alpha\left(X_{I}\right)$.

2. For any trajectory $\tau:[0, T) \mapsto X_{I}$ of $\mathcal{S}, \alpha \circ \tau$ is a trajectory of $\mathcal{T}$.

A simulation relation implies that any time trajectory of $\mathcal{S}$ can be mapped to a trajectory of $\mathcal{T}$ through $\alpha$. However, since $\alpha$ need not be invertible, the converse need not hold: i.e, $\mathcal{T}$ may exhibit time trajectories that are not mapped onto by any trajectory in $\mathcal{S}$.

Let $\mathcal{S}$ and $\mathcal{T}$ be defined by Lipschitz continuous vector fields. The following theorem enables us to check given $\mathcal{S}$ and $\mathcal{T}$, if $\mathcal{T}$ simulates $\mathcal{S}$.

Theorem 2.1. $\mathcal{T}$ simulates $\mathcal{S}$ if the following conditions hold:

1. $Y_{0} \supseteq \alpha\left(X_{0}\right)$.

2. $Y_{I} \supseteq \alpha\left(X_{I}\right)$.

3. $\mathcal{G}(\alpha(\vec{x}))=J_{\alpha} \cdot \mathcal{F}(\vec{x})$, wherein, $J_{\alpha}$ is the Jacobian matrix

$$
\begin{gathered}
J_{\alpha}\left(x_{1}, \ldots, x_{n}\right)=\left[\begin{array}{ccl}
\frac{\partial \alpha_{1}}{\partial x_{1}} & \cdots & \frac{\partial \alpha_{1}}{\partial x_{n}} \\
\vdots & \ddots & \vdots \\
\frac{\partial \alpha_{m}}{\partial x_{1}} & \cdots & \frac{\partial \alpha_{m}}{\partial x_{n}}
\end{array}\right], \\
\text { and } \alpha(\vec{x})=\left(\alpha_{1}(\vec{x}), \cdots, \alpha_{m}(\vec{x})\right), \alpha_{i}: \mathbb{R}^{n} \mapsto \mathbb{R} .
\end{gathered}
$$


Proof. Let $\tau_{x}:[0, T) \mapsto \mathbb{R}^{n}$ be a trajectory over $\vec{x}$ for system $\mathcal{S}$. Note that at any time instant $t \in[0, T), \frac{d \tau_{x}}{d t}=\mathcal{F}(\tau(t))$.

We wish to show that $\tau_{y}(t)=\alpha\left(\tau_{x}(t)\right)$ is a time trajectory for the system $\mathcal{T}$. Since, $\tau_{x}(0) \in X_{0}$, we conclude that $\tau_{y}(0)=\alpha\left(\tau_{x}(0)\right) \in Y_{0}$. Since $\tau_{x}(t) \in X_{I}$ for all $t \in[0, T)$, we have that $\tau_{y}(t)=\alpha\left(\tau_{x}(t)\right) \in Y_{I}$. Differentiating $\tau_{y}$ we get,

$$
\begin{aligned}
\frac{d \tau_{y}}{d t} & =\frac{d \alpha\left(\tau_{x}(t)\right)}{d t}=J_{\alpha} \cdot \frac{d \tau_{x}}{d t}=J_{\alpha} \cdot \mathcal{F}\left(\tau_{x}(t)\right) \\
& =\mathcal{G}\left(\alpha\left(\tau_{x}(t)\right)\right)=\mathcal{G}\left(\tau_{y}(t)\right) .
\end{aligned}
$$

Therefore $\tau_{y}=\alpha \circ \tau_{x}$ conforms to the dynamics of $\mathcal{T}$. By Lipschitz continuity of $\mathcal{G}$, we obtain that $\tau_{y}$ is the unique trajectory starting from $\alpha \circ \tau(0)$.

Theorem 2.1 shows that the condition

$$
\mathcal{G}(\alpha(\vec{x}))=J_{\alpha} \cdot \mathcal{F}(\vec{x})
$$

relating vector fields $\mathcal{F}$ and $\mathcal{G}$ suffices to guarantee that time trajectories (integral curves) of $\mathcal{F}$ are related to those in $\mathcal{G}$ through the map $\alpha$. In differential geometric terms, this condition can be stated as $\mathcal{F}$ is $\alpha$-related to $\mathcal{G}$ [32].

Note that, in general, a trajectory $\tau_{y}(t)=\alpha\left(\tau_{x}(t)\right)$ may exist for a longer interval of time than the interval $[0, T)$ over which $\tau_{x}$ is assumed to be defined.

Theorem 2.2. Let $\mathcal{T}$ simulate $\mathcal{S}$ through a map $\alpha$. If $Y \subseteq Y_{I}$ is a positive invariant set for $\mathcal{T}$ then $\alpha^{-1}(Y) \cap X_{I}$ is a positive invariant set for $\mathcal{S}$.

Proof. Assuming otherwise, let $\tau_{x}$ be a time trajectory that starts from inside $\alpha^{-1}(Y) \cap X_{I}$ and has a time instant $t$ such that $\tau_{x}(t) \notin \alpha^{-1}(Y) \cap X_{I}$. Since we defined time trajectories so that $\tau_{x}(t) \in X_{I}$, it follows that $\tau_{x}(t) \notin \alpha^{-1}(Y)$. As a result, $\alpha\left(\tau_{x}(t)\right) \notin Y$. Therefore, corresponding to $\tau_{x}$, we define a new trajectory $\tau_{y}=\alpha \circ \tau_{x}$ which violates the positive invariance of $Y$. This leads to a contradiction.

Given a constraint $\varphi[\vec{y}]$ that represents an invariant over $Y$, we can translate it back through $\alpha^{-1}$ simply by substituting $\alpha(\vec{x})$ for $\vec{y}$. As a result, $\varphi[\vec{y} \mapsto \alpha(\vec{x})]$ is the corresponding invariant over $X$. An application of this fact is illustrated in Example 1.1, wherein we transfer invariants inferred on the abstract system back in terms of the original system through the map $\alpha$.

Example 2.1. Consider a mechanical system $\mathcal{S}$ expressed in generalized position coordinates $\left(q_{1}, q_{2}\right)$ and momenta $\left(p_{1}, p_{2}\right)$ defined using the following vector field:

$$
F\left(p_{1}, p_{2}, q_{1}, q_{2}\right):\left\langle-2 q_{1} q_{2}^{2},-2 q_{1}^{2} q_{2}, 2 p_{1}, 2 p_{2}\right\rangle
$$

with the initial conditions: $\left(p_{1}, p_{2}\right) \in[-1,1] \times[-1,1] \wedge\left(q_{1}, q_{2}\right):(2,2)$. Using the transformation $\alpha\left(p_{1}, p_{2}, q_{1}, q_{2}\right): p_{1}^{2}+p_{2}^{2}+q_{1}^{2} q_{2}^{2}$, we see that $\mathcal{S}$ is simulated by a linear system $\mathcal{T}$ over $y$, with dynamics given by $\frac{d y}{d t}=0, y(0) \in[16,18]$.

Incidentally, the form of the system $\mathcal{T}$ above indicates that $\alpha$ is an expression for a conserved quantity (in this case, the Hamiltonian) of the system. 
The main goal of this work is to study CoB transformations that "simplify" the system's dynamics either (a) casting a non-algebraic vector field into one defined algebraically or (b) reducing the degree of a given algebraic vector field by means of an abstraction. A special case consists of linearizing CoB transformations that map a non-linear system to one defined by affine dynamics.

Recall that a system $\mathcal{T}$ is algebraic if it is described by a polynomial vector field. Furthermore, $\mathcal{T}$ is affine if it is described by an affine vector field $\frac{d \vec{y}}{d t}=$ $A \vec{y}+\vec{b}$ for an $m \times m$ matrix $A$ and an $m \times 1$ vector $\vec{b}$.

Definition 2.4. Let $\mathcal{S}$ be a (non-linear) system. We say that $\alpha$ is an algebraizing $C o B$ transformation if it maps $\mathcal{S}$ to an algebraic system $\mathcal{T}$.

We say that $\alpha$ is a linearizing CoB transformation if it maps each trajectory of $\mathcal{S}$ to that of an affine system $\mathcal{T}$.

Example 2.2. Consider the vector field $\mathcal{F}$

$$
\frac{d x}{d t}=x^{3}-2 x^{2}+y^{2}+x y, \frac{d y}{d t}=2 x-3 x^{2}+2 y^{3} .
$$

Let $\alpha:(x, y) \rightarrow\left(w_{1}, w_{2}, w_{3}, w_{4}\right)$ be defined as

$$
\alpha(x, y):\left(x, y, x^{2}, y^{2}\right)
$$
$\mathcal{G}:$

We can verify that using $\alpha$, we note that $\mathcal{F}$ is simulated by the vector field

$$
\begin{array}{lll}
\frac{d w_{1}}{d t}=w_{1} w_{3}-2 w_{3}+w_{4}+w_{1} w_{2}, & \frac{d w_{2}}{d t}=2 w_{1}-3 w_{3}+2 w_{2} w_{4} \\
\frac{d w_{3}}{d t}=-4 w_{1} w_{3}+2 w_{3}^{2}+2 w_{1} w_{3}+2 w_{1} w_{4}, & \frac{d w_{4}}{d t}=4 w_{1} w_{2}-6 w_{2} w_{3}+4 w_{4}^{2}
\end{array}
$$

Note that while $\mathcal{F}$ is a cubic vector field over $\mathbb{R}^{2}, \mathcal{G}$ is a quadratic vector field over $\mathbb{R}^{4}$.

Example 1.1 illustrates a linearizing $\mathrm{CoB}$ transformation.

The above definition of an algebraizing or linearizing CoB seems useful, in practice, only if $\alpha$ and $\mathcal{T}$ are already known. We may then use known techniques for reasoning over algebraic systems or affine systems for safely bounding the reachable set of an affine system, given some initial conditions, and transform the result back through $\alpha^{-1}$ to obtain a bound on the reachable set for $\mathcal{S}$.

We now present a technique that searches for a map $\alpha$ to obtain an algebraic system $\mathcal{T}$ that simulates a given system $\mathcal{S}$ through $\alpha$ such that the vector field describing $\mathcal{T}$ is degree bounded by some given degree limit $d>0$. In particular, if the degree limit $d$ is set to 1 , then the resulting transformation $\alpha$ is linearizing.

We ignore the initial condition and invariant, for the time being, and simply focus on obtaining the dynamics of $\mathcal{T}$. In other words, we will search for a map $\alpha:\left(\alpha_{1}, \ldots, \alpha_{m}\right)$ that maps $\mathbb{R}^{n}$ into $\mathbb{R}^{m}$ so that

$$
J_{\alpha}(\vec{x}) \cdot \mathcal{F}(\vec{x})=G(\alpha(\vec{x})) .
$$

Having found such a map, we may find appropriate over-approximate initial and invariance conditions for the simulating system $\mathcal{T}$, so that Definition 2.3 
holds. Specifically, we are interested in finding transformations $\alpha$ that ensure that (a) $G$ is a polynomial vector field and (b) the degrees of polynomials describing $G$ are degree bounded by some given degree limit $d>0$.

\subsection{Multilinear Abstractions through Dimension Copying}

We first show that any polynomial system of ODEs can be abstracted by a multilinear system. However, doing so may require $\alpha$ to have many repeated components wherein $\alpha_{i}(\vec{x})=\alpha_{j}(\vec{x})$ for $i \neq j$. This result is well-known and has been used, for instance, to extend known results on invariant rectangles for multiaffine systems to arbitrary polynomial systems by Ben Sassi et al. [57].

Definition 2.5. A polynomial $p$ is defined to be multilinear if and only if each power-product in $p$ is of the form $x_{1}^{r_{1}} x_{2}^{r_{2}} \cdots x_{n}^{r_{n}}$ wherein each $r_{i}=0$ or 1 .

Example 2.3. As an example, the polynomial $p=2 x_{1} x_{2} x_{3}+x_{1} x_{3}+4 x_{1}-2 x_{2}-1$ is multilinear. On the other hand, the polynomial $q=2 x_{2}^{2}+x_{1}+x_{3}$ is not, owing to the $x_{2}^{2}$ power product.

We first observe that any polynomial ODE may be equivalently written by means of a multilinear system using a suitably defined $\alpha$.

Theorem 2.3. Let $\mathcal{F}$ be a polynomial vector field over $\vec{x} \in \mathbb{R}^{n}$. There is a transformation $\alpha: \mathbb{R}^{n} \rightarrow \mathbb{R}^{m}$, that maps $\mathcal{F}$ to a multilinear system $\mathcal{G}$.

Proof. Let us write $\mathcal{F}(\vec{x}):\left(p_{1}, \ldots, p_{n}\right)$ for multivariate polynomials $p_{1}, \ldots, p_{n}$. We will assume that the vector field $\mathcal{F}$ is not already multi-linear. Therefore, some $p_{j}$ has a power product that is divisible $x_{k}^{r}$ for some $r \geq 2$. The idea is to use $r$ different functions $\alpha_{k, 1}=\alpha_{k, 2}=\cdots=\alpha_{k, r}=x_{k}$ so that in the transformed system the term $x_{k}^{r}$ appears as a multilinear product $y_{k, 1} y_{k, 2} \cdots y_{k, r}$.

In the worst case, the transformation $\alpha$ involves $n \times K$ components, wherein $K=\max \left(\operatorname{degree}\left(p_{1}\right), \ldots\right.$, degree $\left.\left(p_{n}\right)\right)$. Each component $\alpha_{i, k}: x_{i}$ is simply a "copy" of the variable $x_{i}$ that ensures multilinearity of the transformed system.

Example 2.4. Consider the one dimensional system defined by

$$
\frac{d x}{d t}=2 x^{5}+3 x^{2}+x-5 .
$$

We use the transformation $\alpha: \mathbb{R} \rightarrow \mathbb{R}^{5}$ wherein $\alpha_{1}(x)=\alpha_{2}(x)=\cdots=\alpha_{5}(x)=$ $x$. Using this transformation, we derive an abstract system defined by the ODE

$$
\frac{d y_{j}}{d t}=2 y_{1} y_{2} y_{3} y_{4} y_{5}+3 y_{1} y_{2}+y_{1}-5, j=1,2, \ldots, 5 .
$$

Even though there are efficient algorithms for analyzing multi-linear systems [5], the transformation in Theorem 2.3 faces two problems: (a) the dimensionality of the transformed system $\mathcal{T}$ is often forbiddingly large, and (b) 
forgetting the implicit equality relationships between the various dimensions results in a very coarse abstraction, while maintaining these equalities as state invariants of the transformed system may invalidate the special insights into the dynamics multi-linear systems that enable efficient reachability analysis.

\subsection{Independent Transformations}

The rest of this paper, will focus on independent transformations $\alpha:\left(\alpha_{1}, \ldots, \alpha_{N}\right)$ wherein each $\alpha_{i}$ cannot be written as a linear combination of the remaining $\alpha_{j} \mathrm{~S}$ for $j \neq i$. Assuming independence automatically rules out the constructions used in Theorem 2.3.

In general, computing independent transformations $\alpha$ for any given ODE is a hard problem. In this paper, we will focus on solutions that involve searching for an appropriate map $\alpha$, wherein $\alpha$ is specified to be the linear combination of some fixed, finite set of basis functions $g_{1}, \ldots, g_{N}$. The initial basis is assumed to be given to our algorithm by the user. Starting from this initial basis of functions, our algorithm searches for transformations $\alpha$ whose components can be written as linear combinations $\sum_{i=1}^{N} \lambda_{j} g_{j}$.

The basis functions could be specified implicitly as the set of all power products over $\vec{x}$ of degree upto some limit $K>0$ or the set of all power products involving the variables $x_{i}$ and various non-algebraic functions $\sin (z), \cos (z)$ and $e^{z}$ applied to these power products. Having chosen a basis $B=\left\{g_{1}, \ldots, g_{N}\right\}$ for $\alpha$, we will cast the search for the map $\alpha$ as a vector space iteration.

Let $\alpha(\vec{x}):\left(\alpha_{1}(\vec{x}), \ldots, \alpha_{m}(\vec{x})\right)$ be a smooth mapping $\alpha: \mathbb{R}^{n} \mapsto \mathbb{R}^{m}$, wherein each $\alpha_{i}: \mathbb{R}^{n} \mapsto \mathbb{R}$. Recall that $\mathcal{L}_{F}\left(\alpha_{i}(\vec{x})\right)=\left(\nabla \alpha_{i}\right) \cdot \mathcal{F}(\vec{x})$ denotes the Lie derivative of the function $\alpha_{i}(\vec{x})$ w.r.t vector field $\mathcal{F}$.

Lemma 2.1. $J_{\alpha} \cdot \mathcal{F}(\vec{x})=\left(\begin{array}{c}\mathcal{L}_{F}\left(\alpha_{1}(\vec{x})\right) \\ \mathcal{L}_{F}\left(\alpha_{2}(\vec{x})\right) \\ \vdots \\ \mathcal{L}_{F}\left(\alpha_{m}(\vec{x})\right)\end{array}\right)$.

Proof. Recall the definition of the Jacobian matrix $J_{\alpha}$ :

$$
J_{\alpha}\left(x_{1}, \ldots, x_{n}\right)=\left[\begin{array}{ccl}
\frac{\partial y_{1}}{\partial x_{1}} & \cdots & \frac{\partial y_{1}}{\partial x_{n}} \\
\vdots & \ddots & \vdots \\
\frac{\partial y_{m}}{\partial x_{1}} & \cdots & \frac{\partial y_{m}}{\partial x_{n}}
\end{array}\right]=\left[\begin{array}{c}
\nabla \alpha_{1} \\
\vdots \\
\nabla \alpha_{m}
\end{array}\right] .
$$

Therefore, $J_{\alpha} \cdot \mathcal{F}=\left(\begin{array}{c}\left(\nabla \alpha_{1}\right) \cdot(\mathcal{F}) \\ \left(\nabla \alpha_{2}\right) \cdot(\mathcal{F}) \\ \vdots \\ \left(\nabla \alpha_{m}\right) \cdot(\mathcal{F})\end{array}\right)=\left(\begin{array}{c}\mathcal{L}_{F}\left(\alpha_{1}(\vec{x})\right) \\ \mathcal{L}_{F}\left(\alpha_{2}(\vec{x})\right) \\ \vdots \\ \mathcal{L}_{F}\left(\alpha_{m}(\vec{x})\right)\end{array}\right)$.

Note: For the rest of this section, we will fix a vector field $\mathcal{F}$ belonging to a system $\mathcal{S}$ as the original system for which we seek an abstraction and use $\mathcal{L}_{F}$ to denote the Lie derivative according to $\mathcal{F}$. 


\subsection{Vector Space Closure}

We first define the vector spaces that will be used in our search.

Definition 2.6. Let $B=\left\{g_{1}, \ldots, g_{k}\right\}$ be some finite set of functions wherein $g_{i}: \mathbb{R}^{n} \rightarrow \mathbb{R}$ for $n>0$. The vector space spanned by $B$ denoted $\operatorname{Span}(B)$ consists of all functions that are linear combinations of $g_{i}$ :

$$
\operatorname{Span}(B)=\left\{\sum_{i=1}^{k} \lambda_{i} g_{i} \mid \lambda_{i} \in \mathbb{R}\right\}
$$

We assume, without loss of generality, that the elements in $B$ are linearly independent: i.e., no $g_{i} \in B$ can be written as a linear combination of the remaining $g_{j} \in B$, for $j \neq i$.

Let $\mathbf{1}$ represent the constant function $\mathbf{1}(\vec{x})=1, \forall \vec{x} \in \mathbb{R}^{n}$. Given a vector space $V=\operatorname{Span}(B)$, we define the space of power products of $V$ upto a degree limit $d \geq 1$ as

$$
V^{\langle d\rangle}=\operatorname{Span}\left(\left\{g_{i_{1}} \times g_{i_{2}} \times \cdots \times g_{i_{d}} \mid g_{i_{1}}, \ldots, g_{i_{d}} \in B \cup\{\mathbf{1}\}\right\}\right) .
$$

In particular, note that $V^{\langle 1\rangle}=\operatorname{Span}(V \cup\{\mathbf{1}\})$.

Example 2.5. Let $B=\{x, \sin (y)\}$ be our basis set. The vector space $V$ : $\operatorname{Span}(B)$ is given by $\left\{a_{1} x+a_{2} \sin (y) \mid a_{1}, a_{2} \in \mathbb{R}\right\}$. The space $V^{\langle 2\rangle}$ is the set

$$
\left\{a_{0}+a_{1} x+a_{2} \sin (y)+a_{3} x \sin (y)+a_{4} x^{2}+a_{5} \sin ^{2}(y) \mid a_{0}, \ldots, a_{5} \in \mathbb{R}\right\} .
$$

This space is generated by the functions $\mathbf{1}, x, \sin (y), x \sin (y), x^{2}, \sin ^{2}(y)$. It consists of all polynomials of degree at most 2 formed by the functions $x, \sin (y)$. The purpose of adding the function $\mathbf{1}$ is to enable terms of degree 1 and 0 to be considered.

Roughly, the main idea behind our approach is to find a vector space $U$ that satisfies the following closure property:

$$
(\forall f \in U) \mathcal{L}_{F}(f) \in U^{\langle d\rangle} .
$$

In other words, we will search for a vector space $U$, such that taking the Lie derivative of any element of $U$ yields an element in $U^{\langle d\rangle}$. Such a vector space $U$ will be called $d$-closed. Let $U=\operatorname{Span}\left(\left\{h_{1}, \ldots, h_{m}\right\}\right)$ be a $d$ - closed vector space. We will prove that $\alpha:\left(h_{1}, \ldots, h_{m}\right)$ maps the original system $\mathcal{S}$ to an algebraic system $\mathcal{T}$ with a vector field of degree $d$.

Definition 2.7. A vector space $V$ is said to be $d$-closed under the application of Lie derivatives iff $(\forall f \in V) \mathcal{L}_{F}(f) \in V^{\langle d\rangle}$.

In order to check whether a given space $V=\operatorname{Span}(B)$ is $d$-closed, it suffices to verify the property in Definition 2.7 for the elements in $B$. 
Lemma 2.2. A vector space $U=\operatorname{Span}\left(\left\{h_{1}, \ldots, h_{m}\right\}\right)$ be $d$-closed under Lie derivatives if and only if $\mathcal{L}_{F}\left(h_{i}\right) \in U^{\langle d\rangle}$ for $i \in\{1, \ldots, m\}$.

Proof. If $U$ is $d$-closed under Lie derivatives then by definition, the Lie derivatives of its basis elements $h_{i}$ should lie in $U^{\langle d\rangle}$. We will prove the reverse direction. Let $U$ be such that for each basis element $h_{i}$, we have $\mathcal{L}_{F}\left(h_{i}\right) \in U^{\langle d\rangle}$. Any element of $U$ can be written as $f=\sum_{j=1}^{k} a_{j} h_{j}$ for $a_{j} \in \mathbb{R}$. We have $\mathcal{L}_{F}(f)=\sum_{j=1}^{k} a_{j} \mathcal{L}_{F}\left(h_{j}\right)$. Since each $\mathcal{L}_{F}\left(h_{j}\right) \in U^{\langle d\rangle}$, we have that $\mathcal{L}_{F}(f) \in$ $U^{\langle d\rangle}$. This completes the proof.

Next, we relate $d$-closed vector spaces to algebraizing CoB transformations. Let $B=\left\{h_{1}, \ldots, h_{m}\right\}$ and $U=\operatorname{Span}(B)$ be a $d-$ closed vector space. Let $\alpha$ be the map from $\mathbb{R}^{n} \rightarrow \mathbb{R}^{m}$ defined as $\alpha:\left(h_{1}, \ldots, h_{m}\right)$.

Theorem 2.4. The map $\alpha$ formed by the basis elements of a $d$-closed vector field is an algebraizing transformation from the original system $\mathcal{S}$ to a system $\mathcal{T}$ defined by a polynomial vector field of degree at most $d$.

Proof. Since $U$ is $d$-closed, we note that for each $h_{i}$ in the basis of $U$, we have $\mathcal{L}_{F}\left(h_{i}\right) \in U^{\langle d\rangle}$. In other words, we may write $\mathcal{L}_{F}\left(h_{i}\right)$ as a linear combination of power products as shown below:

$$
\mathcal{L}_{F}\left(h_{i}\right): \sum_{j=1}^{K} a_{i j} h_{i, j, 1} \times h_{i, j, 2} \times \cdots \times h_{i, j, d}, \text { wherein } h_{i, j, k} \in B \cup\{\mathbf{1}\}
$$

We define the system $\mathcal{T}$ over variables $y_{1}, \ldots, y_{m}$. We will use variable $y_{i}$ to correspond to $h_{i}(\vec{x})$. The dynamics are obtained as

$$
\frac{d y_{i}}{d t}=\sum_{j=1}^{K} a_{i j} y_{i, j_{1}} \times y_{i, j_{2}} \times \cdots \times y_{i, j_{k}}
$$

by substituting the variable $y_{j}$ wherever the function $h_{j}$ occurs in Equation (1). Let $G$ be the resulting vector field on $\vec{y}$. It is easy to see that (a) $G$ is a polynomial vector field and (b) of degree at most $d$.

From Lemma 2.1 , we note that $J_{\alpha} \cdot \mathcal{F}(\vec{x})=\left(\mathcal{L}_{F}\left(h_{1}\right), \ldots, \mathcal{L}_{F}\left(h_{m}\right)\right)$. We verify that $\left(\mathcal{L}_{F}\left(h_{1}\right), \ldots, \mathcal{L}_{F}\left(h_{m}\right)\right)=G\left(h_{1}(\vec{x}), \ldots, h_{m}(\vec{x})\right)$. This is directly evident from the construction of $G$ from Equation (1). Thus, the key condition (3) of Theorem 2.1 is seen to hold. By finding the right sets $Y_{0}, Y_{I}$ given $\alpha$, we take care of the remaining conditions as well.

Note: The trivial space $V=\operatorname{Span}(\{0\})$ consisting just of the constant function that maps all inputs to $\overrightarrow{0}$ is always $d$-closed. This space yields $\alpha:(0)$ that maps all states $\vec{x}$ to the zero vector. As such, the map $\alpha$ is not very useful in practice for inferring invariants. 
Example 2.6. Consider the ODE from Example 1.1 recalled below:

$$
\begin{aligned}
\frac{d x}{d t} & =x y+2 x \\
\frac{d y}{d t} & =-\frac{1}{2} y^{2}+7 y+1
\end{aligned}
$$

We claim that the vector space $V$ generated by the set of functions $\left\{x, x y, x y^{2}\right\}$ is 1 -closed. To verify, we compute the Lie derivative of a function of the form $c_{1} x+c_{2} x y+c_{3} x y^{2}$ to obtain

$$
c_{1}(x y+2 x)+c_{2}\left(\frac{1}{2} x y^{2}+9 x y+x\right)+c_{3}\left(16 x y^{2}+2 x y\right)
$$

which is seen to belong to $V^{\langle 1\rangle}$. As a result, we obtain the CoB abstraction $\alpha(x, y):\left(x, x y, x y^{2}\right)$ that maps the vector field to an affine vector field (polynomial of degree 1$)$.

The abstract system over $\left(w_{1}, w_{2}, w_{3}\right) \in \mathbb{R}^{3}$ has dynamics given by

$$
\begin{aligned}
\frac{d w_{1}}{d t} & =2 w_{1}+w_{2} \\
\frac{d w_{2}}{d t} & =\frac{1}{2} w_{3}+9 w_{2}+w_{1} \\
\frac{d w_{3}}{d t} & =16 w_{3}+2 w_{2}
\end{aligned}
$$

The mapping between original and abstract system is given by

$$
w_{1} \mapsto x, w_{2} \mapsto x y, w_{3} \mapsto x y^{2} .
$$

\subsection{Finding Closed Vector Spaces}

We will now describe a search technique for finding a map $\alpha$ and the associated abstraction $\mathcal{T}$, such that the dynamics of $\mathcal{T}$ are described by polynomials with degree bound $d$. If $d=1$, the dynamics of $\mathcal{T}$ are affine. The inputs to our search procedure are

1. The original system $\mathcal{S}$ described by a vector field $\mathcal{F}$,

2. The degree limit $d$ for the desired vector field $\mathcal{T}$, and

3. An initial basis $B_{0}=\left\{h_{1}, \ldots, h_{N}\right\}$ of continuous and differentiable functions. We may regard the linear combination

$$
c_{1} h_{1}(\vec{x})+c_{2} h_{2}(\vec{x})+\ldots+c_{N} h_{N}(\vec{x}),
$$

as an ansatz or a template for each component $\alpha_{j}$ of the map $\alpha:\left(\alpha_{1}, \ldots, \alpha_{m}\right)$, that we are searching for. However, we do not fix the number of components $m$ of the transformation $\alpha$, apriori, or guarantee that a non-trivial $\alpha$ (with $m>0$ ) can be found. 
The initial basis $B_{0}$ is often specified as consisting of all power products of the variables in $\vec{x}$ with a given degree limit $M$. This limit $M$ is chosen independent of the limit $d$ for the desired abstraction $\mathcal{T}$.

Our overall approach is to start with the initial vector space $V_{0}: \operatorname{Span}\left(B_{0}\right)$ and iteratively refine $V_{0}$ to construct a sequence of spaces

$$
V_{0} \supseteq V_{1} \supseteq V_{2} \cdots \supseteq V_{k}=V_{k+1}=V^{*}
$$

wherein, (1) $V_{j+1} \subseteq V_{j}$, for $j \in[1, k-1]$, and (2) $V_{k}=V_{k+1}$. The iterative scheme is designed to guarantee that the converged result $V^{*}$ is $d$-closed. If $V^{*}$ has a non-zero basis, then the basis elements of $V^{*}$ form the components of the map $\alpha$ and the abstraction $\mathcal{T}$ whose dynamics have the desired form.

The main step of iteration is to derive $V_{i+1}$ from $V_{i}$. This is performed as follows:

$$
V_{i+1}=\left\{g \in V_{i} \mid \mathcal{L}_{F}(g) \in V_{i}^{\langle d\rangle}\right\} .
$$

In other words, $V_{i+1}$ retains those functions $g \in V_{i}$ whose Lie derivatives also lie inside $V_{i}^{\langle d\rangle}$.

Lemma 2.3. (1) $V_{i+1}$ is a sub-space of $V_{i}$. (2) $V_{i}$ is $d$-closed iff $V_{i}=V_{i+1}$.

Proof. We prove the two parts (1) and (2) as follows.

(1) Since by Eq. (2), $V_{i+1} \subseteq V_{i}$, it suffices to show that $V_{i+1}$ is a vector space. Let $g_{1}, \ldots, g_{k} \in V_{i+1}$. We have that $g_{1}, \ldots, g_{k} \in V_{i}$. Furthermore, since $V_{i}$ is a vector space, any linear combination $g: \sum_{j=1}^{k} \lambda_{j} g_{j} \in V_{i}$. The lie derivative $\mathcal{L}_{F}(g)$ can be written as $\sum_{j=1}^{k} \lambda_{j} \mathcal{L}_{F}\left(g_{j}\right)$. Since $\mathcal{L}_{F}\left(g_{j}\right) \in V_{i}^{\langle d\rangle}$, we have $\mathcal{L}_{F}(g)=\sum_{j=1}^{k} \lambda_{j} \mathcal{L}_{F}\left(g_{j}\right) \in V_{i}^{\langle d\rangle}$. Therefore, by definition $g \in V_{i+1}$ as well. The linear combination of any finite subset of elements from $V_{i+1}$ also belongs to $V_{i+1}$, proving that it is a sub-space of $V_{i}$.

(2) If $V_{i}=V_{i+1}$, it is easy to check that $V_{i}$ satisfies the definition of being $d$ - closed. For the other direction, let us assume that $V_{i}$ is $d$-closed. Then for each $g \in V_{i}$, we have $\mathcal{L}_{F}(g) \in V_{i}^{\langle d\rangle}$. Thus $g \in V_{i+1}$. This proves that $V_{i+1} \supseteq V_{i}$. Combining with the fact that $V_{i+1} \subseteq V_{i}$, we obtain equality.

We now focus on calculating $V_{i+1}$ from $V_{i}$. Let $V_{i}: \operatorname{Span}\left(B_{i}\right)$ for a finite set $B_{i}$. Any element of $V_{i}$ can be represented as $\sum_{h_{j} \in B_{i}} c_{j} h_{j}$ for some multipliers $c_{j}$. The Lie derivative is expressed as $\sum_{h_{j} \in B_{i}} c_{j} \mathcal{L}_{F}\left(h_{j}\right)$. The procedure for calculating $V_{i+1}$ reduces to finding the set of multipliers $\left(c_{1}, \ldots, c_{M}\right)$ where $M=\left|B_{i}\right|$ such that $\sum_{h_{j} \in B_{i}} c_{j} \mathcal{L}_{F}\left(h_{j}\right) \in V_{i}^{\langle d\rangle}$.

The key challenge lies in comparing two elements of the form $\sum_{j} c_{j} \mathcal{L}_{F}\left(h_{j}\right)$ and $\sum_{k} d_{k} g_{k}$, for unknowns $c_{j}$ and $d_{k}$, where $h_{j} \in B_{i}$ and $g_{k} \in V_{i}^{\langle d\rangle}$. If both the functions are polynomials over $\vec{x}$, the comparison is performed by equating the coefficients of corresponding monomials. This is illustrated using the example below:

Example 2.7. Consider once again the ODE from Example 1.1 and 2.6. We seek to find an affine system $\mathcal{T}$ that abstracts this system. Let us consider the 
space $V_{0}$ generated by the basis $B_{0}:\left\{x, y, x y, x^{2}, y^{2}\right\}$ of all degree 2 monomials. Any element in $V_{0}$ can be written as

$$
p\left(c_{1}, \ldots, c_{5}\right): c_{1} x+c_{2} y+c_{3} x y+c_{4} x^{2}+c_{5} y^{2} .
$$

Its Lie derivative is given by

$$
\begin{aligned}
& c_{1}(x y+2 x)+c_{2}\left(-\frac{1}{2} y^{2}+7 y+1\right)+c_{3} x\left(-\frac{1}{2} y^{2}+7 y+1\right) \\
& +c_{3} y(x y+2 x)+c_{4}(2 x)(x y+2 x)+c_{5}(2 y)\left(-\frac{1}{2} y^{2}+7 y+1\right)
\end{aligned}
$$

This can be simplified as

$$
p^{\prime}\left(c_{1}, \ldots, c_{5}\right):\left[\begin{array}{l}
c_{2}+\left(2 c_{1}+c_{3}\right) x+\left(7 c_{2}+2 c_{5}\right) y+\left(c_{1}+9 c_{3}\right) x y+4 c_{4} x^{2}+ \\
\left(14 c_{5}-\frac{1}{2} c_{2}\right) y^{2}+\frac{1}{2} c_{3} x y^{2}+2 c_{4} x^{2} y-c_{5} y^{3}
\end{array}\right] .
$$

We require the Lie derivative to belong to $V^{\langle 1\rangle}=\operatorname{Span}\left(B_{0} \cup\{1\}\right)$. This yields the constraints:

$$
\left(\exists d_{0}, d_{1}, \ldots, d_{5}\right)(\forall x, y) d_{0}+d_{1} x+d_{2} y+d_{3} x y+d_{4} x^{2}+d_{5} y^{2}=p^{\prime}\left(c_{1}, \ldots, c_{5}\right)
$$

We use the lemma that two polynomials are identical iff their coefficients on corresponding power-products are. This yields the following system of linear equations:

$$
\begin{aligned}
& c_{2}=d_{0}, 2 c_{1}+c_{3}=d_{1}, 7 c_{2}+2 c_{5}=d_{2}, c_{1}+9 c_{3}=d_{3}, \\
& 4 c_{4}=d_{4}, 14 c_{5}-\frac{1}{2} c_{2}=d_{5}, c_{3}=0,2 c_{4}=0, c_{5}=0
\end{aligned}
$$

Eliminating $d_{0}, \ldots, d_{5}$, we obtain the constraints $c_{3}=c_{4}=c_{5}=0$. The new basis $B_{1}$ is $\{x, y\}$.

On the other hand, if the basis $B_{i}$ involves non-polynomials (trigonometric or exponential functions), then encoding equality by matching up coefficients of syntactically identical terms is incomplete: i.e, not all solutions can be found by equating coefficients of matching terms. In general, deciding if expressions involving trigonometric functions are identically zero is undecidable ${ }^{2}$ In practice, we may continue to handle trigonometric functions using the same syntactic matching technique that is complete for polynomials. If a $d$-closed basis is discovered this way, then it may be used to derive a valid abstraction. On the other hand, the process may be unable to find a vector space starting from the initial set of functions even if one such exists.

Example 2.8. Consider a simple example with the ODE

$$
\frac{d x}{d t}=\sin (x+y), \quad \frac{d y}{d t}=x+y .
$$

Consider the space $V$ spanned by the basis

$$
B=\{x, y, \sin (x), \sin (y), \cos (x), \cos (y)\} .
$$

\footnotetext{
${ }^{2}$ This follows from Richardson's theorem [42].
} 
To check if $V$ is 3 -closed, we note that any element of $V$ can be written as

$$
c_{1} x+c_{2} y+c_{3} \sin (x)+c_{4} \sin (y)+c_{5} \cos (x)+c_{6} \cos (y) .
$$

Its Lie derivative can be written as

$$
\begin{gathered}
c_{1} \sin (x+y)+c_{2}(x+y)+c_{3} \cos (x) \sin (x+y)+c_{4} \cos (y)(x+y) \\
-c_{5} \sin (x) \sin (x+y)-c_{6} \sin (y)(x+y)
\end{gathered} .
$$

Our goal is to check if the Lie derivative belongs to $V^{\langle 3\rangle}$. We note that a syntactic check for membership yields the constraints $c_{1}=c_{3}=c_{5}=0$. On the other hand, substituting the trigonometric identity

$$
\sin (x+y) \equiv \sin x \cos y+\sin y \cos x
$$

we may indeed verify that the Lie derivative of any element of $V$ belongs to $V^{\langle 3\rangle}$. This yields a degree 3 algebraization given by $\alpha(x, y):(x, y, \sin (x), \sin (y), \cos (x), \cos (y))$ with the abstract system having the dynamics

$$
\begin{aligned}
\frac{d w_{1}}{d t} & =w_{3} w_{6}+w_{4} w_{5} \\
\frac{d w_{2}}{d t} & =w_{1}+w_{2} \\
\frac{d w_{3}}{d t} & =w_{3} w_{5} w_{6}+w_{5}^{2} w_{4} \\
\frac{d w_{4}}{d t} & =w_{6} w_{1}+w_{6} w_{2} \\
\frac{d w_{5}}{d t} & =-w_{3}^{2} w_{6}-w_{3} w_{4} w_{5} \\
\frac{d w_{6}}{d t} & =-w_{4} w_{1}-w_{4} w_{2}
\end{aligned}
$$

Here $w_{1}, \ldots, w_{6}$ correspond to the components of the map $\alpha$ above.

Theorem 2.5. Given an initial vector space $V_{0}$ and vector field $\mathcal{F}$, the iterative procedure using Eqn. (2) converges in finitely many steps to a subspace $V^{*} \subseteq V_{0}$. Let $\alpha_{1}, \ldots, \alpha_{m}$ be the basis functions that generate $V^{*}$.

1. The transformation $\alpha:\left(\alpha_{1}, \ldots, \alpha_{m}\right)$ generated by the basis functions of the final vector space leads to an abstract system whose dynamics are described by polynomials of degree at most $d$.

2. A limited form of completeness holds: Let $U \subseteq V_{0}$ be a subspace that is $d$-closed. It follows that $U \subseteq V^{*}$. In other words, if a d-closed CoB transformation exists whose functions are part of the initial space $V_{0}$ then it is also a part of the final result $V^{*}$.

Proof. Let us represent the iterative sequence as

$$
V_{0} \supseteq V_{1} \supseteq V_{2} \cdots
$$

The convergence of the iteration follows from the observation that if $V_{i+1} \subset V_{i}$, the dimension of $V_{i+1}$ is at least one less than that of $V_{i}$. Since $V_{0}$ is finite dimensional, the number of iterations is upper bounded by the number of basis functions in $V_{0}$. 
The first statement follows directly from Theorem 2.4.

The next statement is proved by induction. Specifically, we prove by induction that $U \subseteq V_{i}$ for each $i$. The base case is true since $U \subseteq V_{0}$ by assumption.

Next, we show that if $U \subseteq V_{i}$ then $U \subseteq V_{i+1}$. This follows from Eq. 2 since for each $p \in U$, we have $p \in V_{i}$ and $\mathcal{L}_{F}(p) \in U^{\langle d\rangle}$. This gives us $\mathcal{L}_{F}(p) \in V_{i}^{\langle d\rangle}$. Therefore, $p \in V_{i+1}$.

As a result, we prove by induction that $U \subseteq V_{i}$ for each $i$. This also means that $U \subseteq V^{*}$.

Note that it is possible for the converged result $V^{*}$ to be trivial. i.e, it is generated by the constant function $\mathbf{1}$.

Example 2.9. Consider the van der Pol oscillator whose dynamics are given by

$$
\dot{x}=y, \dot{y}=\mu\left(y-\frac{1}{3} y^{3}-x\right) .
$$

Our search for polynomials $(\mu=1)$ of degree up to 20 did not yield a non-trivial transformation.

For a trivial system, the resulting affine system $\mathcal{T}$ is $\frac{d y}{d t}=0$ under the map $\alpha(\vec{x})=0$. Naturally, this situation is not quite interesting but will often result, depending on the system $\mathcal{S}$ and the initial basis chosen $V_{0}$. Section 2.7 discusses common situations where the vector space $V^{*}$ obtained as the result is guaranteed to be non-trivial.

\subsubsection{Complexity}

For simplicity, we analyze the time complexity bounds when we search for a 1-closed subspace of the initial space $V_{0}$.

There are two ways of representing vector spaces over polynomials: (a) through an explicit list of the basis elements, or alternatively, (b) using a parameteric polynomial form over $n$ variables:

$$
V(\vec{c}, \vec{x}): \sum c_{\alpha} \vec{x}^{\alpha}
$$

wherein $c_{\alpha}$ is a parameter corresponding to the monomial $\vec{x}^{\alpha}$, and an associated set of constraints $A \vec{c}=0$ over $\vec{c}$. The former is often called a "generator" representation of the vector space, whereas the latter is a "constraint" represenation.

We also note that the steps involved in this computation are remarkably similar to the so-called Karr's algorithm used for generating linear equality invariants for programs originally studied by M. Karr in 1976 [28, 37].

Borrowing ideas from Muller-Olm and Seidl [37], our implementation of this iteration alternates between both the representations of the vector space during the process of computation. At the start of each iteration, we represent $V_{i}$ as a list of polynomials that form its basis. Next, we construct a generic element of $V_{i}$ as a parameteric linear combination of the basis elements:

$$
p(\vec{c}, \vec{x}): \sum_{p_{j} \in V_{i}} c_{j} p_{j}
$$


We compute constraints $A \vec{c}=0$ that are enforcing that the Lie derivative of $p$ belongs back to $V_{i}$. This system is often rank deficient (or else $\vec{c}=0$ and we obtain the trivial result). We compute a set of basis vectors for the nullspace of $A$. This immediately yields a basis for $V_{i+1}$.

The worst-case time complexity of the overall procedure is decomposed into two parts: (a) the worst-case time complexity of each iteration and (b) the number of iterations. For an ODE with $n$ variables, let us start with an initial monomial basis that involves all terms of degree upto $d$. This yields the dimension of the initial vector space $\operatorname{dim}\left(V_{0}\right)=O\left(n^{d}\right)$. This also yields an upper bound on the number of iterations.

Each iteration involves computing the Lie derivative of each of the basis elements in the current vector space $V_{i}$, forming the system $A \vec{c}=0$ and extracting the nullspace for $A$. The latter can be performed through a Gaussian elimination procedure, involving $O\left(\operatorname{dim}\left(V_{i}\right)^{3}\right)$ cost.

Combining, we obtain an overall complexity of $O\left(n^{4 d}\right)$ wherein $n$ is the number of variables in the system and $d$ is the maximum degree of monomials in the original basis $V_{0}$. In our evaluation (see Section 4), we observe two important features that mitigate the high worst-case complexity: (a) our iterations converge in very few iterations even for large initial basis, and (b) the dimensions of the vector spaces reduces considerably yielding a much smaller dimension for the converged result in comparison with the original vector space.

\subsection{Strong and Constant Scale Consecution}

The notion of "strong" consecution, "constant scale" consecution and "polynomial scale" consecution were defined for equality invariants of differential equations in our previous work [55] and subsequently expanded upon by Matringe et al. [34] using the notion of morphisms. We now show that the techniques presented in this section can capture these notions, ensuring that all the systems handled by the techniques presented in our previous work [55] can be handled by the techniques here (but not vice-versa).

Definition 2.8. A function $f$ satisfies the strong scale consecution requirement for a vector field $\mathcal{F}$ iff $\mathcal{L}_{F}(f)=0$. In other words, $f$ is a conserved quantity.

Similarly, $f$ satisfies the constant scale consecution iff $\exists \lambda \in \mathbb{R}, \mathcal{L}_{F}(f)=\lambda f$.

The following theorem is a corollary of Theorem 2.5 and shows that the ideas presented in this section can capture the notion of strong and constant scale consecution without requiring quantifier elimination, solving an eigenvalue problem [55] or finding roots of a univariate polynomial [34].

Theorem 2.6. The result of the iteration $V^{*}$ starting from an initial space $V_{0}$ contains all the strong and constant scale invariant functions in $V_{0}$.

Proof. This is a direct consequence of Theorem 2.5 by noting that for a constant scale consecuting function $f$, the subspace $U \subseteq V_{0}$ spanned by $f$ is closed under Lie derivatives. 
Furthermore, if such functions exist in $V_{0}$ the result after convergence $V^{*}$ is guaranteed to be a non-trivial vector space (of positive dimension). Finally, constant scale and strong scale functions can be extracted by computing the affine equality invariants of the linear system $\mathcal{T}$ that can be extracted from $V^{*}$.

\subsubsection{Stability}

We briefly address the issue of deducing stability (or instability) of a system $\mathcal{S}$ using an abstraction to a system $\mathcal{T}$. Since $\alpha$ satisfies the identity

$$
\mathcal{G}(\alpha(\vec{x}))=J_{\alpha} \cdot \mathcal{F}(\vec{x}) .
$$

Every equilibrium of $\mathcal{S}(\mathcal{F}(\vec{x})=0)$ maps onto an equilibrium of $\mathcal{T}(\mathcal{G}(\vec{x})=0)$, but not vice-versa. Furthermore, the map $\alpha(\vec{x})=(\mathbf{0}, \ldots, \mathbf{0})$ is an abstraction from any non-linear system to one with an equilibrium at origin. Therefore, unless restrictions are placed on $\alpha$, we are unable to draw conclusions on liveness properties for $\mathcal{S}$ based on $\mathcal{T}$. If $\alpha$ has a continuous inverse, then $\mathcal{T}$ is topologically diffeomorphic to $\mathcal{S}$ [35]. This allows us to correlate equilibria of $\mathcal{T}$ with those of $\mathcal{S}$. The preservation of stability under mappings of state variables has been studied by Vassilyev and Ul'yanov [61]. We are currently investigating restrictions that will allow us to draw conclusions about liveness properties of $\mathcal{S}$ from those of $\mathcal{T}$.

The issue of stability preserving maps between continuous and hybrid systems was recently addressed by the work of Prabhakar et al. [47].

\subsection{Affine CoB Abstraction: Existence}

We will now focus on the special case of $\mathrm{CoB}$ transformations that lead to linear abstractions of the form $\frac{d \vec{w}}{d t}=A \vec{w}$ (and affine abstractions of the form $\left.\frac{d \vec{w}}{d t}=A \vec{w}+\vec{b}\right)$.

Let $\mathcal{S}$ be a non-linear system over $\vec{x}$ that has a CoB transformation $\alpha: \mathbb{R}^{n} \rightarrow$ $\mathbb{R}^{m}$ with $m>0$ that maps to a linear system $\frac{d \vec{w}}{d t}=A \vec{w}$.

Lemma 2.4. The system $\mathcal{S}$ has $m$ conserved quantities given by the components of the vector valued function $e^{-t A} \alpha(\vec{x})$.

Proof. Our goal is to prove that the Lie derivative of each component of $e^{-t A} \alpha(\vec{x})$ equals zero. Since $\alpha$ is a linearizing $\mathrm{CoB}$, we have $\mathcal{L}_{F}(\alpha(\vec{x}))=A \alpha(\vec{x})$.

The Lie derivative of $e^{-t A} \alpha(\vec{x})$ is given by

$$
e^{-t A} \mathcal{L}_{F}(\alpha(\vec{x}))+\partial_{t} e^{-t A} \alpha(\vec{x})=e^{-t A} A \alpha(\vec{x})-e^{-t A} A \alpha(\vec{x})=0 .
$$

Thus we see that the Lie derivative of $e^{-t A} \alpha(\vec{x})$ vanishes. Therefore, each component of $e^{-t A} \alpha(\vec{x})$ is a conserved quantity.

Conversely, whenever the original system $\mathcal{S}$ has conserved quantities, it trivially admits the linearization $\frac{d \vec{w}}{d t}=0$ using a transformation $\alpha$ that is formed by its conserved quantity. 
Theorem 2.7. A system $\mathcal{S}$ has an independent, linearizing CoB transformation $\alpha: \mathbb{R}^{n} \mapsto \mathbb{R}^{m}$ if and only if it has $m$ linearly independent conserved quantities.

The theorem extends to affine $\mathrm{CoB}$ transformations that yield abstract systems of the form $\frac{d \vec{w}}{d t}=A \vec{w}+\vec{b}$. While conservative mechanical and electromagnetic systems naturally have conserved quantities (eg., conservation of momentum, energy, charge, mass), many systems encountered are dissipative. Such cases are handled by extending the approach presented here to differential inequality abstractions [52].

Furthermore, even in a setting where conservative quantities exist, the advantages of searching for a $\mathrm{CoB}$ transformation as opposed to directly searching for a conserved quantity from an ansatz are not clear at a first glance. The advantage of the techniques presented here lies in the fact that existing techniques that search for conserved quantities focus for the most part on finding polynomial conserved quantities. Whereas, searching for a $\mathrm{CoB}$ transformation allows us to implicitly obtain conserved quantities that may involve exponentials, sines and cosines in addition to polynomial conserved quantities by focusing purely on reasoning with vector spaces generated by polynomials.

Example 2.10. We observed the following conserved quantity for the system in Example 1.1

$$
\begin{aligned}
& \left(\frac{e^{-9 t}}{51}+\frac{1}{102}(50+7 \sqrt{51}) e^{(-9+\sqrt{51}) t}+\frac{1}{102}(50-7 \sqrt{51}) e^{-(9+\sqrt{51}) t}\right) x+ \\
& \left(-\frac{1}{102} e^{-9 t-(9+\sqrt{51}) t}\left(\begin{array}{l}
7 e^{9 t}-\sqrt{51} e^{9 t}-14 e^{(9+\sqrt{51}) t}+ \\
7 e^{9 t+(-9+\sqrt{51}) t+(9+\sqrt{51}) t}+ \\
\sqrt{51} e^{9 t+(-9+\sqrt{51}) t+(9+\sqrt{51}) t}
\end{array}\right)\right) x y+ \\
& \left(\frac{1}{204} e^{-9 t-(9+\sqrt{51}) t}\left(e^{9 t}-2 e^{(9+\sqrt{51}) t}+e^{9 t+(-9+\sqrt{51}) t+(9+\sqrt{51}) t}\right)\right) x y^{2}
\end{aligned}
$$

This is one of the three conserved quantities obtained by computing $e^{-t A} \alpha(\vec{x})$, where

$$
\alpha:\left(x, x y, x y^{2}\right) \text { and } A=\left(\begin{array}{ccc}
2 & 1 & 0 \\
1 & 9 & \frac{1}{2} \\
0 & 2 & 16
\end{array}\right) .
$$

We are unaware of techniques that can directly generate such conserved quantities.

\subsection{Change-of-Bases For Improved Flowpipe Construction}

Theorem 2.2 already establishes a key motivation behind our study of CoB abstractions for ODEs: any property established for the abstract system can, in fact, be transferred back to the original system. Motivating example 1.1 illustrates this property for the simple running example. We now briefly examine another application of this idea to improve the process of flowpipe construction for verifying (time bounded) reachability properties of continuous and hybrid systems. 

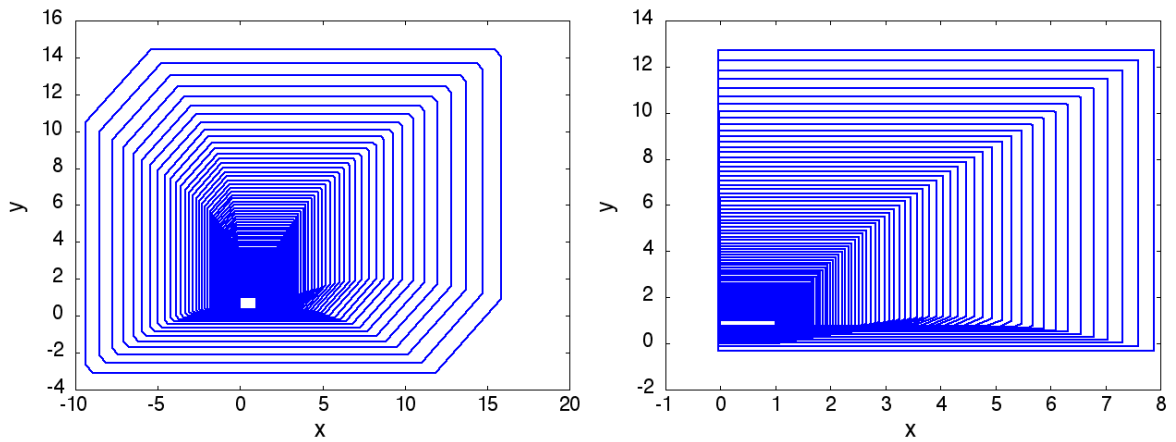

Figure 2: Comparing the flowpipes constructed for Flow* for the system in Example 1.1. (Left) The original 2 state model and (Right) The augmented model including the original system and the linear abstraction.

Given an initial set of states $X_{0}$ for a system of differential equations $\dot{\vec{x}}=$ $f(\vec{x})$, the goal of flowpipe construction is to find approximations for the set $X_{t}$ of reachable states at time $t$, where $t \in[0, T]$ for a given time horizon $T>0$. Approaches to flowpipe construction have been derived for the specific case of linear (affine) hybrid systems $[23,31,21,25,22]$ and for nonlinear continuoustime and hybrid systems $[62,3,58,9,48,39,10,6]$. The key question is whether $\mathrm{CoB}$ abstractions can help in the process of flowpipe construction. We will investigate this using a tool called Flow* that the author has been involved in [10]. Flow* ${ }^{*}$ represents approximations of the solution of a differential equation using a Taylor model [6]. Taylor models are used to approximate smooth functions over a compact set by means of a Taylor polynomial and an interval that overapproximates the error. Integration using Taylor model was originally proposed by Berz and Makino [7], and further refined by many in the interval analysis community. Flow* implements a form of Taylor model integration that employs adaptive step sizing and choice of polynomial degrees during the integration process. It also takes into account mode invariants that constraint the evolution of the ODE, and can handle multimodal hybrid dynamics specified by means of switching surfaces and reset maps $[9,10]$.

Let $\mathcal{G}$ be an affine system that simulates our original nonlinear system $\mathcal{F}$ through a $\mathrm{CoB}$ transformation $\vec{w}=\alpha(\vec{x})$. We created an augmented system $\mathcal{F G}$ over the system of variables $(\vec{x}, \vec{w})$ linked by the constraint $\vec{w}=\alpha(\vec{x})$, that is added as an "invariant" to constrain the state-space of the system. The augmented dynamics include the original dynamics $\frac{d \vec{x}}{d t}=f(\vec{x})$ and the affine system $\frac{d \vec{w}}{d t}=A \vec{w}$. On one hand, augmenting the original system through the affine abstraction does not change the set of trajectories for the original system. In fact, it is easy to see that the $\vec{w}$ variables are superfluous, in this sense. However, in the context of an interval analysis, the augmentation helps because it adds back extra constraints that must be respected along all trajectories of 
the system.

Example 2.11. Consider, once again, the system from Ex. 1.1, recalled below:

$$
\dot{x}=x y+2 x, \quad \dot{y}=-\frac{1}{2} y^{2}+7 y+1
$$

and the abstraction discovered through the transformation $\alpha:(x, y) \mapsto\left(w_{1}, w_{2}, w_{3}\right)$ wherein $\alpha_{1}(x, y)=x, \alpha_{2}(x, y)=x y$ and $\alpha_{3}(x, y)=x y^{2}$ and the affine dynamics:

$$
\dot{w}_{1}=2 w_{1}+w_{2}, \quad \dot{w}_{2}=w_{1}+9 w_{2}+\frac{1}{2} w_{3}, \dot{w_{3}}=2 w_{2}+16 w_{3}
$$

The augmented system has variables $\left(x, y, w_{1}, w_{2}, w_{3}\right)$ and its dynamics are given by simply combining the original system dynamics with the abstraction, above. We also relate the variables by adding the invariants $w_{1}=x \wedge w_{2}=x y \wedge w_{3}=$ $x y^{2}$. The initial conditions $(x, y) \in[0,1] \times[0,1]$ for the original system translate to $\left(w_{1}, w_{2}, w_{3}\right) \in[0,1]^{3}$ for the additional variables.

Figure 2 compares the results of running Flow* for a time horizon $T=0.4$. Flow* analyzes the original system 0.8 seconds on a Macbook air laptop with $1.8 \mathrm{GHz}$ Intel $(\mathrm{tm})$ processor and $8 \mathrm{~GB}$ RAM, running OSX 10.9. The augmented system takes 78.5 seconds to run on the same system, yielding a more accurate result. For instance, the augmented flowpipe correctly infers that all reachable states belong to the first quadrant $x \geq 0, y \geq 0$. Furthermore, it yields a tighter overall range for the given time horizon.

This example demonstrates that even though augmenting the original system using a $\mathrm{CoB}$ abstraction does not modify the trajectories of the original system, it can potentially improve the precision of flowpipe construction techniques that rely on interval analysis. Section 4 compares the performance of the Flow* tool on the original versus the augmented system for more examples.

\section{Abstractions for Discrete and Hybrid Systems}

In this section, we will discuss how the techniques of the previous sections can be extended to find $\mathrm{CoB}$ transformations of purely discrete programs. In particular, our focus will be on transforming loops in programs to infer abstractions that are of a simpler form. Our presentation will first focus on simple loops consisting of a single location. The combination of loops with multiple locations and continuous dynamics will be handled in the subsequent section.

\subsection{Transition System Models}

We will first define transition system models and the action of CoB transformations on these models. Let $\vec{x} \in X$ represent real valued system variables, where $X \subseteq \mathbb{R}^{n}$. Transition systems will form our basic models for loops in programs [33]. The process of transforming a given imperative program to a transition system representation is related to the idea of control flow graph (CFG) construction, that is implemented in most compilers [36]. 


$$
\begin{aligned}
\vec{x} & :(x, y, k) \\
L & :\left\{\ell_{0}\right\} \\
\mathcal{T} & :\left\{\begin{array}{l}
t_{1}:\left(\ell_{0}, \ell_{0}, G_{1}, F_{1}\right), \\
t_{2}:\left(\ell_{0}, \ell_{0}, G_{2}, F_{2}\right)
\end{array}\right\} \\
X_{0}: & \{(x, y, k) \mid x=y=0 \wedge k>0\} . \\
G_{1}: & \{(x, y, k) \mid y<k\} \\
F_{1}(x, y, k): & \left(x+y^{2}, y+1, k\right)
\end{aligned}
$$

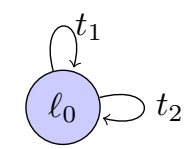

Figure 3: Transition system model for the loop in Example 1.2.

Definition 3.1. A transition system $\Pi$ is defined by a tuple $\left\langle X, L, \mathcal{T}, X_{0}, \ell_{0}\right\rangle$, wherein,

1. $X \subseteq \mathbb{R}^{n}$ represents the continuous state-space. We will denote the system variables by $\vec{x} \in \mathbb{R}^{n}$.

2. $L$ denotes a finite set of locations.

3. $\mathcal{T}$ represents a finite set of transitions. Each transition $t_{j} \in \mathcal{T}$ is a tuple $\left\langle\ell_{j}, m_{j}, G_{j}, F_{j}\right\rangle$, where

- $\ell_{j} \in L$ is the pre-location of the transition, and $m_{j} \in L$ is the postlocation.

- $G_{j} \subseteq \mathbb{R}^{n}$ is the guard condition on the system variables $\vec{x}$.

- $F_{j}: \mathbb{R}^{n} \rightarrow \mathbb{R}^{n}$ is the update function.

4. $X_{0} \subseteq X$ represents the possible set of initial values and $\ell_{0} \in L$ represents the starting location.

Example 3.1. Figure 3 shows an example of a transition system derived from a simple program that computes the sum of the first $k$ squares. The transition system consists of a single location $\ell_{0}$, transitions $t_{1}:\left(\ell_{0}, \ell_{0}, G_{1}, F_{1}\right)$ and $t_{2}$ : $\left(\ell_{0}, \ell_{0}, G_{2}, F_{2}\right)$.

A state of the transition system is a tuple $\sigma:\langle\ell, \vec{x}\rangle$ where $\ell$ is the current location and $\vec{x} \in X$ are the values of the continuous variables.

A run is a finite or infinite sequence of states

$$
\sigma_{0} \stackrel{t_{0}}{\rightarrow} \sigma_{1} \stackrel{t_{1}}{\rightarrow} \cdots \rightarrow \sigma_{j} \stackrel{t_{j}}{\longrightarrow} \sigma_{j+1} \cdots,
$$

where each $\sigma_{j}:\left(\ell_{j}, \vec{x}_{j}\right)$ is a state and $t_{j}$ a transition, satisfying the following conditions: 
1. The starting state $\sigma_{0}:\left(\ell_{0}, \vec{x}_{0}\right)$ is initial: i.e., $\ell_{0}$ is the initial location of $\Pi$ and $\vec{x}_{0} \in X_{0}$.

2. The state $\sigma_{i+1}:\left(\ell_{i+1}, \vec{x}_{i+1}\right)$ is related to the state $\sigma_{i}:\left(\ell_{i}, \vec{x}_{i}\right)$ in the following way:

(a) The transition $t_{i} \in \mathcal{T}$ is of the form $\left(\ell_{i}, \ell_{i+1}, G_{i}, F_{i}\right)$, leading from $\ell_{i}$ to $\ell_{i+1}$.

(b) The valuation $\vec{x}_{i}$ of the continuous variables satisfy the guard $G_{i}$ and the valuation $\vec{x}_{i+1}$ is obtained by executing the assignments in $F_{i}$ on $\vec{x}_{i}$ :

$$
\vec{x}_{i} \in G_{i} \text { and } \vec{x}_{i+1}=F_{i}\left(\vec{x}_{i}\right) .
$$

A special class of "simple loop" transition systems that have a single location.

Definition 3.2. A transition system $\Pi$ is called a simple loop if it has a single location: i.e., $L=\{\ell\}$. All transitions of a simple loop are self-loops around this location $\ell$.

The transition system in Example 3.1 is a simple loop. It consists of a single location. In general, simple loops can have multiple transitions that "loop" around this single location.

We will now discuss the pre-image operator FPRE induced by a transition. Let $g(\vec{x})$ be some function over the state variables and $t:(\ell, m, G, F)$ be a transition.

Definition 3.3. The functional pre-image $\operatorname{FPRE}(g, t)$ is defined as $g(F(\vec{x}))$.

Note: The standard precondition operator works over assertions over the state variables, involving computing the pre-image using $F$ and computing the intersection of the result with the guard. The functional precondition defined here is defined over functions $g(\vec{x})$ over the state variables.

Example 3.2. Consider the transition

$$
\left.t:(\ell, m, G, F) \text {, wherein } G:\{(x, y) \mid x \geq y\}, F(x, y):\left(x^{2}, y^{2}-x^{2}\right)\right) .
$$

The functional pre-image of the function $g(x, y): x+y$, denoted $\operatorname{FPRE}(x+y, \rho)$, is given by

$$
\operatorname{FPRE}(x+y, t):\left(x^{2}\right)+\left(y^{2}-x^{2}\right)=y^{2} .
$$

To contrast with the standard pre-condition operator, which applies to assertions over states, let us consider the assertion $x+y \geq 0$. We have

$$
\operatorname{PRE}(x+y \geq 0, \rho): y^{2} \geq 0 \wedge x \geq y .
$$

We now show that FPRE is a linear operator over functions. 
Lemma 3.1. For any transition $t$ and functions $g_{1}, g_{2}, g$ over $\vec{x}$, we have $\operatorname{FPRE}\left(g_{1}+g_{2}, t\right)=\operatorname{FPRE}\left(g_{1}, t\right)+\operatorname{FPRE}\left(g_{2}, t\right)$ and further, $\operatorname{FPRE}(\lambda g)=\lambda \operatorname{FPRE}(g)$ for any $\lambda \in \mathbb{R}$.

Proof. Proof follows by directly applying Def. 3.3.

Let us consider any run of the transition system

$$
r: \sigma_{0} \stackrel{t_{0}}{\rightarrow} \sigma_{1} \rightarrow \cdots \rightarrow \sigma_{i} \stackrel{t_{i}}{\rightarrow} \sigma_{i+1} \cdots .
$$

Let $t_{i}:\left(\ell_{i}, \ell_{i+1}, G_{i}, F_{i}\right)$ denote the transition between $\sigma_{i}:\left(\ell_{i}, \vec{x}_{i}\right)$ and $\sigma_{i+1}$ : $\left(\ell_{i+1}, \vec{x}_{i+1}\right)$. Finally, let $g(\vec{x})$ be any function over the state varaibles of the transition system.

Lemma 3.2. The following identity holds for all successive pairs of states $\left(\ell_{i}, \vec{x}_{i}\right) \stackrel{t_{i}}{\rightarrow}\left(\ell_{i+1}, \vec{x}_{i+1}\right)$ encountered in a run of the transition system and for all functions $g(\vec{x})$ :

$$
\operatorname{FPRE}\left(g(\vec{x}), t_{i}\right) \equiv g\left(\vec{x}_{i+1}\right)
$$

Proof. We may write $h(\vec{x})=g(F(\vec{x}))$. We know that $\vec{x}_{i+1}=F\left(\vec{x}_{i}\right)$. Therefore, $g\left(\vec{x}_{i+1}\right)=g\left(F\left(\vec{x}_{i}\right)\right)=h\left(\vec{x}_{i}\right)$.

We will now discuss change-of-basis abstractions for transition systems. The discussion will focus on defining change-of-basis abstractions for simple loops, which are represented by a transition system with a single location $\ell$ (Cf. Definition 3.2). The subsequent sections will extend this concept to arbitrary transition systems.

\subsection{CoB Abstractions For Simple Loops}

Consider a simple loop $\Pi$ over $\vec{x} \in \mathbb{R}^{n}$ with a single location $\ell$, transitions $\left\{t_{1}, \ldots, t_{k}\right\}$, and initial condition $X_{0}$. We seek to abstract $\Pi$ with another simple loop $\Xi$ over $\vec{y} \in \mathbb{R}^{d}$ with a single location $m$, transitions $\left\{t_{1}^{\prime}, \ldots, t_{k}^{\prime}\right\}$ and initial condition $Y_{0}$.

Definition 3.4. Simple loop $\Xi$ is a $\mathrm{CoB}$ abstraction of $\Pi$ iff there is a continuous function $\alpha: \mathbb{R}^{n} \rightarrow \mathbb{R}^{d}$ such that

1. The initial condition $Y_{0} \supseteq \alpha\left(X_{0}\right)$,

2. For each transition $t_{i}:\left(\ell, \ell, G_{i}, F_{i}\right)$ in $\Pi$, there is a corresponding transition $t_{i}^{\prime}:\left(m, m, G_{i}^{\prime}, F_{i}^{\prime}\right)$ in $\Xi$ such that

(a) $G_{i}^{\prime} \supseteq \alpha\left(G_{i}\right)$,

(b) $\forall \vec{x} F_{i}^{\prime}(\alpha(\vec{x}))=\alpha\left(F_{i}(\vec{x})\right)$.

We will now present an example of $\mathrm{CoB}$ abstraction for simple loops. 
Example 3.3. Consider the simple loop from Example 3.1 (also Fig. 3). We note that the map

$$
\alpha: \mathbb{R}^{3} \rightarrow \mathbb{R}^{4}, \text { where } \alpha:\left(x, y, k, y^{2}\right),
$$

yields an abstract transition system $\Xi$ over variables $\vec{w}:\left(w_{1}, w_{2}, w_{3}, w_{4}\right)$. Informally, the variables $\left(w_{1}, w_{2}, w_{3}, w_{4}\right)$ are placeholders for the expressions $\left(x, y, k, y^{2}\right)$, respectively. The resulting transition system $\Xi$ is

$$
\begin{aligned}
\vec{w} & :\left(w_{1}, \ldots, w_{4}\right) \\
L & :\{m\} \\
\mathcal{T} & :\left\{t_{1}^{\prime}:\left(m, m, G_{1}^{\prime}, F_{1}^{\prime}\right), t_{2}^{\prime}:\left(m, m, G_{2}^{\prime}, F_{2}^{\prime}\right)\right\} \\
X_{0} & : w_{1}=w_{2}=w_{4}=0 \wedge w_{3} \geq 1 \\
G_{1}^{\prime} & :\left\{\vec{w} \mid w_{2}<w_{3}\right\} \\
G_{2}^{\prime} & :\left\{\vec{w} \mid w_{2} \geq w_{3}\right\} \\
F_{1}^{\prime}(\vec{w}) & :\left(w_{1}+w_{4}, w_{2}+1, w_{3}, w_{4}+2 w_{2}+1\right) \\
F_{2}^{\prime}(\vec{w}) & : \vec{w}
\end{aligned}
$$

The various requirements laid out in Definition 3.4 can be easily verified. We will verify the requirement for $F_{1}^{\prime}: F_{1}^{\prime}(\alpha(x, y, k))=\alpha\left(F_{1}(x, y, k)\right)$, as follows:

$$
\begin{aligned}
F_{1}^{\prime}(\alpha(x, y, k)) & =F_{1}^{\prime}\left(x, y, k, y^{2}\right)=(\underbrace{x+y^{2}}_{w_{1}+w_{4}}, \underbrace{y+1}_{w_{2}+1}, \underbrace{k}_{w_{3}}, \underbrace{y^{2}+2 y+1}_{w_{4}+2 w_{2}+1}) . \\
& =\alpha\left(x+y^{2}, y+1, k\right)=\alpha\left(F_{1}(x, y, k)\right)
\end{aligned} .
$$

The definition of $\mathrm{CoB}$ abstraction immediately admits the following key theorem.

Theorem 3.1. For any run

$$
\sigma_{0}:\left(\ell, \vec{x}_{0}\right) \stackrel{t_{0}}{\longrightarrow}\left(\ell, \vec{x}_{1}\right) \stackrel{t_{1}}{\longrightarrow}\left(\ell, \vec{x}_{2}\right) \stackrel{t_{2}}{\longrightarrow} \cdots
$$

the corresponding sequence of $\Xi$-states

$$
\gamma_{0}:\left(m, \alpha\left(\vec{x}_{0}\right)\right) \stackrel{t_{0}^{\prime}}{\longrightarrow}\left(m, \alpha\left(\vec{x}_{1}\right)\right) \stackrel{t_{1}^{\prime}}{\longrightarrow}\left(m, \alpha\left(\vec{x}_{2}\right)\right) \stackrel{t_{2}^{\prime}}{\longrightarrow} \cdots,
$$

is a run of $\Xi$.

Proof. Proof uses the property that whenever the move $\left(\ell, \vec{x}_{j}\right) \stackrel{t_{j}}{\rightarrow}\left(\ell, \vec{x}_{j+1}\right)$ is possible in $\Pi$ then the move $\left(m, \alpha\left(\vec{x}_{j}\right)\right) \stackrel{t_{j}^{\prime}}{\longrightarrow}\left(m, \alpha\left(\vec{x}_{j+1}\right)\right)$ is possible in $\Xi$.

Let $t_{j}$ be described by the guard $G_{j}$ and the functional update $F_{j}$. Likewise, let $t_{j}^{\prime}$ be described by $G_{j}^{\prime}$ and $F_{j}^{\prime}$. We note that $\alpha\left(G_{j}\right) \subseteq G_{j}^{\prime}$. Since $\vec{x}_{j}$ satisfies the guard of $t_{j}, \alpha\left(\vec{x}_{j}\right)$ satisfies that of $t_{j}^{\prime}$. The state obtained after the transition is given by $\vec{x}_{j+1}^{\prime}=F^{\prime}\left(\alpha\left(\vec{x}_{j}\right)\right) \alpha\left(F\left(\vec{x}_{j}\right)\right)=\alpha\left(\vec{x}_{j+1}\right)$.

We have proved that whenever the move $\left(\ell, \vec{x}_{j}\right) \stackrel{t_{j}}{\rightarrow}\left(\ell, \vec{x}_{j+1}\right)$ is possible in $\Pi$ then the move $\left(m, \alpha\left(\vec{x}_{j}\right)\right) \stackrel{t_{j}^{\prime}}{\rightarrow}\left(m, \alpha\left(\vec{x}_{j+1}\right)\right)$ is possible in $\Xi$. The rest of the proof extends this to trace containment through induction over prefixes of the traces. 
As a direct consequence, we may state a theorem that corresponds to Theorem 2.2 for the case of vector fields. Given a formula $\varphi[\vec{x}]$, we use $[[\varphi]]$ to denote the set of valuations to $\vec{x}$ that satisfy $\varphi$.

Theorem 3.2. Let $[[\varphi]]$ be an invariant set for the abstract system $\Xi$. Then, $\alpha^{-1}(\varphi)$ is an invariant of the original system $\Pi$.

Proof. First, we note from Theorem 3.1 that if $(\ell, \vec{x})$ is reachable in $\Pi$ then $(m, \alpha(\vec{x}))$ is reachable in $\Xi$. Since $\varphi$ is an invariant for $\Xi$, we have $(m, \alpha(\vec{x})) \in$ $[[\varphi]]$. Therefore for any reachable state $(\ell, \vec{x})$ in $\Pi$, we have $(\ell, \vec{x}) \in \alpha^{-1}([[\varphi]])$. Thus $\alpha^{-1}([[\varphi]])$ is an invariant set for $\Xi$.

Given an invariant $\varphi[\vec{y}]$ for $\Xi$ in the form of an assertion, the invariants for the original system are obtained simply by substituting $\alpha(\vec{x})$ in the place of $\vec{y}$ in $\varphi$.

Example 3.4. Consider the transition system $\Pi$ from Example 3.1 and its abstraction $\Xi$ in Example 3.3. We note that $\Xi$ has affine guards and updates. Therefore, we may use a standard polyhedral analysis tool to compute invariants over $\Xi[15,26,53]$. Some of the invariants obtained include

$$
\begin{aligned}
& 13 w_{4} \leq 9 w_{1}+24 w_{2} \wedge 7 w_{4} \leq 6 w_{1}+11 w_{2} \wedge 4 w_{1}+7 w_{2}-7 w_{4}+11 w_{3} \geq 11 \\
& 2 w_{1}+3 w_{2}-3 w_{4}+4 w_{3} \geq 4 \wedge w_{4} \leq 2 w_{1}+w_{2} \wedge 3 w_{4} \leq w_{1}+12 w_{2} \\
& 9-w_{1}-3 w_{2}+3 w_{4}-9 w_{3} \leq 0 \wedge w_{2} \geq 0 \wedge 1 \leq w_{3} \wedge w_{2}-w_{3} \leq 0
\end{aligned}
$$

By substituting $w_{1} \mapsto x, w_{2} \mapsto y, w_{3} \mapsto k, w_{4} \mapsto y^{2}$ on these invariants, we conclude invariants for the original system. For instance, we conclude facts such as

$$
13 y^{2}-24 y-9 x \geq 0 \wedge 7 y^{2}-11 y-6 \geq 0 \wedge 11 k-7 y^{2}+7 y+4 x \geq 11
$$

The goal, once again, is to find an abstraction $\alpha$ and an abstract system $\Xi$ starting from a description of the system $\Pi$. Furthermore, we require that the update functions $F_{j}^{\prime}$ in $\Xi$ are all polynomials whose degrees are smaller than some given limit $d>0$. In particular, if we set $d=1$, we are effectively requiring all the updates in $\Xi$ to be affine functions over $\vec{y}$.

Our strategy will be to find a map $\alpha: \mathbb{R}^{n} \rightarrow \mathbb{R}^{k}$. For convenience, we will write $\alpha$ as $\left(\alpha_{1}, \ldots, \alpha_{k}\right)$, wherein each component function $\alpha_{j}: \mathbb{R}^{n} \rightarrow$ $\mathbb{R}$. Let $V$ be the vector space spanned by the components of $\alpha$, i.e, $V=$ $\operatorname{Span}\left(\left\{\alpha_{1}, \ldots, \alpha_{k}\right\}\right)$. Our goal will be to ensure that for each transition $t$ in $\Pi$,

$$
\forall \vec{x}, \operatorname{FPRE}\left(\alpha_{i}(\vec{x}), t\right) \in V^{\langle d\rangle} .
$$

Let $V$ be a vector space that satisfies Eq. (3) for each transition $t$ in $\Pi$. We will say that the space $V$ is $d$-closed w.r.t $\Pi$. 
Theorem 3.3. Let $V: \operatorname{Span}\left(g_{1}, \ldots, g_{k}\right)$ be d-closed w.r.t $\Pi$ for continuous functions $g_{1}, \ldots, g_{k}$. The map $\alpha:\left(g_{1}, \ldots, g_{k}\right)$ is a CoB transformation defining an abstract system $\Xi$, wherein each transition of $\Xi$ has a polynomial update function involving polynomials of degree at most $d$.

Proof. We construct the abstract system $\Xi$ with variables $w_{1}, \ldots, w_{k}$ representing the functions $g_{1}, \ldots, g_{k}$ in the original system. $\Xi$ has a single location $m$ and for each transition $t_{i} \in \Pi$, we construct a corresponding transition $t_{i}^{\prime} \in \Xi$ as follows.

Let $G_{i}, F_{i}$ be the guard and update sets for $t_{i}$. The guard set for $t_{i}^{\prime}$ is given by $\alpha\left(G_{i}\right)$ or an over-approximation thereof. Likewise, the update $F_{i}^{\prime}$ for $t_{i}^{\prime}$ is derived as follows. We note that

$$
\operatorname{FPRE}\left(g_{j}, t_{i}\right)=\sum_{r} c_{r_{1}, r_{2}, \ldots, r_{k}} g_{1}^{r_{1}} g_{2}^{r_{2}} \cdots g_{k}^{r_{k}},
$$

wherein $0 \leq r_{1}+r_{2}+\ldots+r_{k} \leq d$. The corresponding update for $w_{j}$ in the abstract system is given by

$$
F_{i}^{\prime}\left(w_{j}\right)=\sum_{r} c_{r_{1}, r_{2}, \ldots, r_{k}} w_{1}^{r_{1}} w_{2}^{r_{2}} \cdots w_{k}^{r_{k}} .
$$

Note that each function $F_{i}^{\prime}\left(w_{j}\right)$ is a polynomial of degree at most $d$ over $w_{1}, \ldots, w_{k}$.

Since the operator FPRE used to define the closure in Eq. (3) is a linear operator (Cf. Lemma 3.1), we may check the closure property for a given vector space $V$ by checking if its basis functions satisfy the property.

Lemma 3.3. The vector space $V: \operatorname{Span}\left(\left\{g_{1}, \ldots, g_{k}\right\}\right)$ is d-closed w.r.t $\Pi$ iff for each basis element $g_{i}$ of $V$, and for each transition $t$ in $\Pi, \operatorname{FPRE}\left(g_{i}, t\right) \in V^{\langle d\rangle}$.

Proof. For the non-trivial direction, let $V$ be a space where for each basis element $g_{i}$ of $V$, and for each transition $t$ in $\Pi, \operatorname{FPRE}\left(g_{i}, t\right) \in V^{\langle d\rangle}$. An arbitrary element $g \in V$ can be written as a linear combination of its basis elements: $g=\sum_{j} \lambda_{j} g_{j}$. We have $\operatorname{FPRE}(g, t)=\sum_{j} \lambda_{j} \operatorname{FPRE}\left(g_{j}, t\right)$ from Lemma 3.1. Since $\operatorname{FPRE}\left(g_{j}, t\right) \in V^{\langle d\rangle}$, which is a vector space itself, we have that $\operatorname{FPRE}(g, t)$ is a linear combination of elements in $V^{\langle d\rangle}$ and thus $\operatorname{FPRE}(g, t) \in V^{\langle d\rangle}$. Thus $V$ is $d$-closed.

Example 3.5. Once again, consider the system $\Pi$ in Example 3.1 and the map $\alpha:\left(x, y, k, y^{2}\right)$ from Example 3.3. The components of this map are the functions $\alpha_{1}: x, \alpha_{2}: y, \alpha_{3}: k$, and $\alpha_{4}: y^{2}$. We may verify that the vector space $V: \operatorname{Span}\left(\left\{x, y, k, y^{2}\right\}\right)$ satisfies the closeure property in Eq. (3) for $d=1$. The table below shows the results of applying FPRE on each of the basis elements.

\begin{tabular}{|l|l|l|}
\hline Basis function $g_{j}$ & $\operatorname{FPRE}\left(g_{j}, t_{1}\right)$ & $\operatorname{FPRE}\left(g_{j}, t_{2}\right)$ \\
\hline$x$ & $x+y^{2}$ & $x$ \\
$y$ & $y+1$ & $y$ \\
$k$ & $k$ & $k$ \\
$y^{2}$ & $y^{2}+2 y+1$ & $y^{2}$ \\
\hline
\end{tabular}


Thus, $\operatorname{FPRE}\left(g_{j}, t_{k}\right)$ belongs to $V^{\langle 1\rangle}=\operatorname{Span}\left(\left\{1, x, y, k, y^{2}\right\}\right)$.

Searching for Abstractions: The procedure for finding abstractions is identical to that used for vector fields with the caveat that closure under Lie-derivative is replaced by closure under $\operatorname{FPRE}\left(\cdot, t_{j}\right)$ for every transition $t_{j}$ in the system. The procedure takes as input an initial basis of functions $B_{0}$ and iteratively refines the vector space $V_{i}: \operatorname{Span}\left(B_{i}\right)$ by removing all the functions that do not satisfy the closure property.

Example 3.6. Consider the system $\Pi$ in Example 3.1 and the initial basis consisting of all monomials of degree at most 2 over variables $x, y, k$. We obtain the basis $B_{0}:\left\{x, y, k, x^{2}, y^{2}, k^{2}, x y, y k, x k\right\}$ and the space $V_{0}: \operatorname{Span}\left(B_{0}\right)$. An element of $V_{0}$ can be written as

$$
p:\left[\begin{array}{c}
c_{1} x+c_{2} y+c_{3} k+c_{4} x^{2}+c_{5} y^{2}+c_{6} k^{2} \\
+c_{7} x y+c_{8} y k+c_{9} x k
\end{array}\right]
$$

We consider the transition $t_{1}$ with update $F_{1}(x, y, k):\left(x+y^{2}, y+1, k\right)$. Transition $t_{2}$ is ignored as its update is simply the identity relation. We have $\operatorname{FPRE}\left(p, t_{1}\right)$ as

$$
\operatorname{FPRE}\left(p, t_{1}\right):\left[\begin{array}{c}
\left(c_{2}+c_{5}\right)+\left(c_{1}+c_{7}\right) x+\left(c_{2}+2 c_{5}\right) y+\left(c_{3}+c_{8}\right) k+c_{4} x^{2}+ \\
\left(c_{1}+c_{5}+c_{7}\right) y^{2}+c_{6} k^{2}+c_{7} x y+c_{7} y^{3}+c_{4} y^{4}+2 c_{4} x y^{2}+ \\
c_{8} y k+c_{9} x k+c_{9} y^{2} k
\end{array}\right]
$$

The "overflow" terms $c_{7} y^{3}, c_{4} y^{4}, c_{9} y^{2} k$ immediately yield the constraints $c_{4}=$ $c_{7}=c_{9}=0$. The refined basis is $B_{1}:\left\{x, y, k, y^{2}, k^{2}, y k\right\}$. The iterative process converges with $V_{1}: \operatorname{Span}\left(B_{1}\right)$ yielding a linearization.

Complexity: The process of computing the vector space iterates and the overall complexity are quite the same as for the case of ODEs 2.6.1. The major change is that the computation of Lie derivatives is replaced with computing preconditions.

\subsection{Abstractions for General Transition Systems}

Thus far, we have presented $\mathrm{CoB}$ abstractions for simple loops consisting of a single location. The ideas seamlessly extend to systems with multiple locations with a few generalizations that will be described in this section.

Let $\Pi$ be a system with a set of locations $L=\left\{\ell_{1}, \ldots, \ell_{k}\right\}$ and transitions $\mathcal{T}$. We will assume that $|L| \geq 2$ so that the system is no longer a simple loop. The main idea behind change-of-bases $(\mathrm{CoB})$ transformations for systems with multiple locations is to allow a different map for each location. In other words, the abstraction is defined by a maps $\alpha_{\ell}(\vec{x})$ for each location $\ell \in L$.

The maps for two different locations $\ell_{1}$ and $\ell_{2}$ are of the type $\alpha_{\ell_{1}}: \mathbb{R}^{n} \rightarrow$ $\mathbb{R}^{m_{1}}$ and $\alpha_{\ell_{2}}: \mathbb{R}^{n} \rightarrow \mathbb{R}^{m_{2}}$. In general, we may assume that $m_{1} \neq m_{2}$. This discrepancy can be remedied by padding each $\alpha_{\ell_{i}}$ with extra components that 
three locations $m_{1}, m_{2}, m_{3}$ corresponding to $\ell_{1}, \ell_{2}, \ell_{3}$, respectively and three transitions $t_{1}^{\prime}, t_{2}^{\prime}$ and $t_{3}^{\prime}$ corresponding to $t_{1}, t_{2}$ and $t_{3}$ in $\Pi$. The guards and updates of the transition $t_{1}^{\prime}$ are

$$
\begin{aligned}
G_{1}^{\prime} & :\left\{\left(w_{0}, \ldots, w_{8}\right) \mid w_{8}+w_{6}-w_{3} \leq 100\right\} \\
F_{1}^{\prime} & : \quad\left(w_{0}, w_{1}+w_{2}, w_{3}, w_{1}-w_{2}+w_{6}, w_{4}+2 w_{5}+w_{7},-w_{1}+w_{2}+w_{8}, 0,0,0\right)
\end{aligned}
$$

We verify the key condition that ensures that $t_{1}^{\prime}$ is an abstraction of $t_{1}$ :

$$
\alpha_{\ell_{2}}\left(F_{1}(x, y, z)\right)=F_{1}^{\prime}\left(\alpha_{\ell_{1}}(x, y, z)\right) \text {. }
$$

The LHS $\alpha_{\ell_{2}}\left(F_{1}(x, y, z)\right)=\alpha_{\ell_{2}}(x+z x-z y, y+z y-z x, z)$ is given by

$$
\left(z^{2}, z x+z y, z, y+z y-z x, x^{2}+2 x y+y^{2}, x+z x-z y, 0,0,0\right) .
$$

The RHS $F_{1}^{\prime}\left(\alpha_{\ell_{1}}(x, y, z)\right)=F_{1}^{\prime}\left(z^{2}, y z, x z, z, y^{2}, x y, y, x^{2}, x\right)$ is given by

$$
\left(z^{2}, x z+y z, z, y-z x+z y, y^{2}+2 x y+x^{2}, x+z x-z y, 0,0,0\right) .
$$

The identity of LHS and RHS is thus verified.

Our goal once again is to search of a collection of transformations $\alpha_{\ell}$, for each $\ell \in L$ such that the resulting system is described by polynomial updates of degree at most $d$. The case where $d=1$ corresponds to affine updates. Once again, we generalize the notion of a $d$-closed vector space. Consider a collection of vector spaces $V_{\ell}: \operatorname{Span}\left(B_{\ell}\right)$ for each location $\ell \in L$.

Definition 3.6. We say that the collection $V_{\ell}, \ell \in L$ is $d$-closed for transition system $\Pi$ if and only if for each transition $t_{j}:\left\langle\ell_{\text {pre }}, \ell_{\text {post }}, G_{j}, F_{j}\right\rangle$ and for each element $p \in V_{\text {post }}$, we have $\operatorname{FPRE}\left(p, t_{j}\right) \in V_{\text {pre }}^{\langle d\rangle}$.

The notion of $d$-closed vector spaces can be related to CoB transformations and resulting abstractions whose updates are defined by means of polynomials of degree at most $d$.

Theorem 3.4. Let $V_{\ell}, \ell \in L$ be a collection of vector spaces that are $d$-closed for a system $\Pi$. The basis elements of $V_{\ell}$ yields a collection of maps $\alpha_{\ell}, \ell \in L$ that relate $\Pi$ to a $C o B$ abstraction $\Xi$. The update maps of $\Xi$ are all polynomials of degree at most $d$.

Example 3.8. Consider the transition system described in Example 3.7 and Figure 4. We wish to discover an affine abstraction for this system automatically. Starting from the initial collection of vector spaces that maps each location to the space of all polynomials of degree at most 2 over $x, y, z$, we obtain the transformations $\alpha_{\ell_{1}}, \alpha_{\ell_{2}}, \alpha_{\ell_{3}}$ described in the same example. This yields an abstract system over variables $w_{0}, \ldots, w_{8}$. 


\subsection{Combining Discrete and Continuous Systems}

As a final step, we extend our approach to hybrid systems that combine discrete and continuous dynamics. We define hybrid systems briefly and extend the results from Sections 2 and 3 to address hybrid systems.

Definition 3.7. A hybrid system consists of a discrete transition system $\Pi$ : $\left\langle X, L, \mathcal{T}, X_{0}, \ell_{0}\right\rangle$ and a mapping that associates each location $\ell_{i} \in L$ with a continuous subsystem $\mathcal{S}_{i}:\left\langle\mathcal{F}_{i}, X_{i}\right\rangle$ over the state-space $X$, consisting of a vector field $\mathcal{F}_{i}$ and location invariant $X_{i}$.

A state $\sigma$ of the hybrid system consists of a tuple $\langle\ell, \vec{x}, t\rangle$ where $\ell \in L$ is the current location, valuations to the continuous variables $\vec{x} \in X$ and the current time $t \geq 0$.

Given a time $\delta \geq 0$, we write $\langle\ell, \vec{x}, T\rangle \underset{\delta}{\rightsquigarrow}\langle\ell, \vec{y}, T+\delta\rangle$ to denote that starting from state $\langle\ell, \vec{x}, T\rangle$ the hybrid system flows continuously according to the continuous subsystem $\mathcal{S}_{\ell}$ corresponding to the location $\ell$. Likewise, we write $\langle\ell, \vec{x}, T\rangle \stackrel{t_{j}}{\longrightarrow}\left\langle\ell^{\prime}, \vec{x}^{\prime}, T\right\rangle$ to denote a jump between two states upon taking a discrete transition $t_{j}$ from $\ell$ to $\ell^{\prime}$. Note that no time elapses upon taking a jump.

A run $R$ of the hybrid system is given by a countable sequence of alternating flows (evolution according to the ODE inside a location) and jumps (discrete transition to a different location) starting from an initial state:

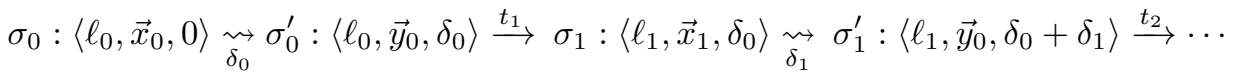

To avoid Zenoness, we require that the summation of the dwell times in the individual modes $\sum_{j=0}^{\infty} \delta_{j}$ diverges.

We now define $\mathrm{CoB}$ abstractions for hybrid systems. Our definitions simply combine aspects of the definition for transition systems 3.5 and continuous systems 2.3.

A $\mathrm{CoB}$ abstraction of the hybrid system is obtained through a collection of maps $\alpha_{\ell_{1}}, \ldots, \alpha_{\ell_{k}}$ corresponding to the locations $\ell_{1}, \ldots, \ell_{k}$ of the hybrid system. It is assumed that by padding with $0 \mathrm{~s}$, we obtain each $\alpha_{\ell_{i}}$ as a function $\mathbb{R}^{n} \rightarrow$ $\mathbb{R}^{m}$.

Definition 3.8. A system $\Xi$ is a $\mathrm{CoB}$ abstraction of $\Pi$ through a collection of maps $\alpha_{\ell_{1}}, \ldots, \alpha_{\ell_{k}}$ each of the type $\mathbb{R}^{n} \rightarrow \mathbb{R}^{m}$, corresponding to locations $\ell_{1}, \ldots, \ell_{k}$, iff

1. $\Xi$ has locations $m_{j}$ corresponding to $\ell_{j} \in L$ for $1 \leq j \leq k$, and transitions $t_{i}^{\prime}$ corresponding to transition $t_{i} \in \mathcal{T}$. Each location $m_{j}$ in $\Xi$ has an associated continuous system $\mathcal{T}_{j}$.

2. For each corresponding location pair $\ell_{j}, m_{j}$, the system $\mathcal{T}_{j}$ is a $\mathrm{CoB}$ abstraction of $\mathcal{S}_{j}$ through the transformation $\alpha_{\ell_{j}}$.

3. For each transition $t_{i}:\left\langle\ell_{\text {pre }}, \ell_{\text {post }}, G_{i}, F_{i}\right\rangle$ in $\Pi$, the corresponding transition $t_{i}^{\prime}:\left\langle m_{\text {pre }}, m_{\text {post }}, G_{i}^{\prime}, F_{i}^{\prime}\right\rangle$ are such that 
(a) $m_{\text {pre }}$ and $m_{\text {post }}$ correspond to $\ell_{\text {pre }}$ and $\ell_{\text {post }}$, respectively,

(b) $G_{i}^{\prime} \supseteq \alpha_{\ell_{p r e}}\left(G_{i}\right)$,

(c) $(\forall \vec{x}) F_{i}^{\prime}\left(\alpha_{\ell_{\text {pre }}}(\vec{x})\right)=\alpha_{\text {post }}\left(F_{i}(\vec{x})\right)$.

Once again, we focus on searching for an abstraction $\Xi$ of a given hybrid system wherein the continuous abstraction for each location and that of each transition is expressed by means of polynomials degree bounded by some fixed bound $d$. The case where the bound is $d=1$ specifies an affine hybrid abstraction $\Xi$. We translate this into a $d$-closure condition for vector spaces. Consider a collection of vector spaces $V_{\ell}: \operatorname{Span}\left(B_{\ell}\right)$ for each location $\ell \in L$.

Definition 3.9. We say that the collection $V_{\ell}, \ell \in L$ is $d$-closed for transition system $\Pi$ if and only if

1. For each location $\ell \in L$, the corresponding vector space $V_{\ell}$ is $d$-closed w.r.t to the vector field $\mathcal{F}_{\ell}$ defining the continuous subsystem $\mathcal{S}_{\ell}$.

2. For each transition $t_{j}:\left\langle\ell_{\text {pre }}, \ell_{\text {post }}, G_{j}, F_{j}\right\rangle$ and for each element $p \in V_{\text {post }}$, we have $\operatorname{FPRE}\left(p, t_{j}\right) \in V_{\text {pre }}^{\langle d\rangle}$.

Once again, the approach for finding a $d$-closed collection $V_{\ell}, \ell \in L$ starts from an initial basis $V_{\ell}^{(0)}$ at each location $\ell$ and refines the basis. Two types of refinements are applied (a) refinement of $V_{\ell}$ to enforce closure w.r.t the Lie derivative of its basis elements for the vector field $\mathcal{F}_{\ell}$ and (b) refinement of $V_{m}$ w.r.t a transition $t:\langle\ell, m, G, F\rangle$ incoming at location $m$.

Abstracting Guard and Invariant Sets: We finally mention that a key difficulty for hybrid systems involves the process of abstracting guards and invariant sets. For instance, suppose the original system has a simple switching guard of the form $x \leq c$, it can be transformed by the abstraction process into a more complicated (nonlinear) guard on the resulting abstract hybrid system with affine dynamics. Similarly, polyhedral mode invariants can become general semi-algebraic invariants for the abstract system. The solution to this problem involves over-approximating guards and invariants through polyhedral sets. This over-approximation can however be quite coarse, introducing many spurious behaviors and also hard to compute, in general.

\section{Implementation and Evaluation}

We have implemented the ideas described in this paper to derive affine abstractions for (a) continuous systems described by ODEs with polynomial righthand sides, (b) discrete systems with assignments that have polynomial RHS and (b) hybrid systems with polynomial ODEs and discrete transition updates. Our approach takes as inputs the system description, a degree limit $k>0$ that is used to construct the initial basis. Starting from this initial basis, our approach iteratively applies refinement until convergence. Upon convergence, we print the basis inferred along with the resulting abstraction. 

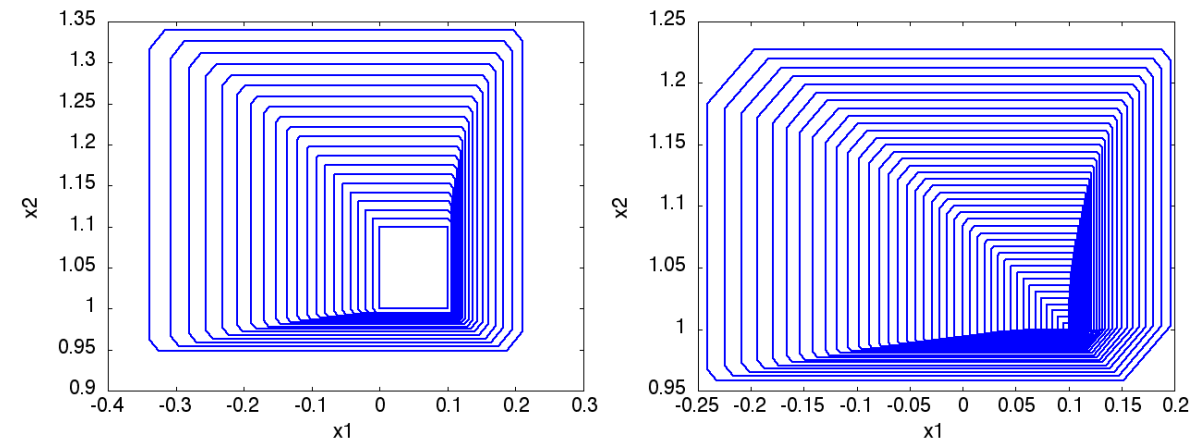

Figure 5: Comparing the flowpipes constructed for Flow* for the system in Toda-2 lattice. (Left) The original 6 state model and (Right) The augmented model with 12 state variables including the original system and the linear abstraction.

Currently, our implementation does not abstract the guard sets of the transitions and the invariant sets of the ODEs. However, once the basis is inferred using quantifier elimination techniques (which is quite expensive in practice) $[12,13,18]$ or techniques such as SOS programming [41]. Our implementation currently relies on manual translation of invariant and guard assertions into the new basis to form the abstract transition system.

If a non-trivial abstraction is discovered by our iterative scheme, we may use a linear invariant generator on the resulting affine system to infer invariants that relate to the original transition system.

\subsection{Continuous Systems}

We first describe experimental results obtained for continuous systems described by ODEs. Table 1 summarizes the results on continuous system benchmarks. We collected nearly 15 benchmark systems and ran our implementation to search for a linearizing CoB transformation. We report on the degree of the monomials in the initial basis, time taken to converge and the number of polynomials in the final basis that form the transformation to the abstract system.

Trivial Transformations Found: Some of the benchmarks attempted resulted in trivial final transformations. Examples include the well-known FitzhughNagumo neuron model, the vanderpol oscillators and similar small but complex systems that are known to be non-integrable. There is as yet no characterization of systems that admit a non-trivial CoB transformation to an affine system.

We now highlight some of the interesting results, while summarizing all benchmarks in Table 1.

Toda Lattice with Boundary Particles: The Toda lattice models an infinite array of point particles such that the position and velocity of the $n^{\text {th }}$ particle 
are affected by its neighbours the $(n-1)^{t h}$ and $(n+1)^{t h}$ particle for $n \in \mathbb{Z}^{3}$. We consider a finite version of this lattice with 2 fixed boundary particles that are constrained to have a fixed position and zero velocity and $K$ particles in the middle. The dynamics for $K=2$ non-fixed particles are given by position variables $y_{1}, y_{2}$, velocities $v_{1}, v_{2}$ and extra state variables $u_{1}, u_{2}$ to model the interaction with neighbours.

$$
\begin{array}{rlll}
\frac{d x_{1}}{d t}=v_{1} & \frac{d v_{1}}{d t}=v_{1}\left(u_{1}-u_{2}\right) & \frac{d u_{1}}{d t}=-v_{1} \\
\frac{d x_{2}}{d t} & =v_{2} \frac{d v_{2}}{d t}=v_{2} u_{2} & \frac{d u_{2}}{d t} & =v_{1}-v_{2}
\end{array}
$$

In addition, we add time $t$ as a variable to the model with dynamics $\frac{d t}{d t}=1$. Our approach initialized with polynomials of degree 2 discovers a basis with 10 polynomials:

$$
\begin{aligned}
& w_{1}:-2 v_{2}-2 v_{1}-u_{2}^{2}+2 x_{1} u_{1}+x_{2}^{2}, \\
& w_{2}:-2 v_{2}-2 v_{1}-u_{2}^{2}+u_{1} u_{2}+x_{2} u_{1}+x_{1} u_{2}+x_{1} u_{1}+x_{1} x_{2} \\
& w_{3}:-2 v_{2}-2 v_{1}+2 u_{1} u_{2}+2 x_{2} u_{2}+2 x_{2} u_{1}+x_{2}^{2}, w_{4}: u_{1}+x_{1}, \\
& w_{5}: 2 v_{2}+2 v_{1}+u_{1}^{2}+u_{2}^{2}, w_{6}: u_{2}+x_{2}-x_{1}, w_{7}: t, \\
& w_{8}: u_{1} t+x_{1} t, w_{9}: u_{2} t+u_{1} t+x_{2} t, w_{10}: t^{2}
\end{aligned}
$$

The resulting abstract system has linear dynamics given by:

$$
\frac{d w_{j}}{d t}=0,1 \leq j \leq 6, \frac{d w_{7}}{d t}=1, \frac{d w_{8}}{d t}=w_{4}, \frac{d w_{9}}{d t}=w_{4}+w_{6}, \frac{d w_{10}}{d t}=2 w_{7}
$$

Results for larger instances are reported in Table 1.

Once again, we augment the original system with six additional variables $w_{1}, \ldots, w_{6}$, ignoring time dependent variables $w_{7}, \ldots, w_{10}$. The initial set $X_{0}$ is given by

$$
x_{1} \in[0,0.1], x_{2} \in[1,1.1], v_{1} \in[-0.1,0], v_{2} \in[0,0.1],\left(u_{1}, u_{2}\right) \in[0,0.1]^{2}
$$

Figure 5 contrasts the original flowpipe for time horizon $T=2$. The original model has 6 state variables and Flow* analyzes this model in 64.5 seconds. The augmented system has 12 state variables and requires 336.5 seconds for Flow* flowpipe construction. However, the figure clearly shows that the augmented system's flowpipe establishes a tighter range over the values of the state variables $\left(x_{1}, x_{2}\right)$ over the time horizon when compared to the original system's flowpipe.

Quadratic Fermi-Pasta-Ulam-Tsingou System: Consider a system considered by Fermi et al. [20]. The system consists of a chain of particles at positions $x_{1}, \ldots, x_{N}$ with fixed boundary particles $x_{0}=0$ and $x_{N+1}=N+1$. The dynamics are given by

$$
\frac{d^{2} x_{i}}{d t^{2}}=\left(x_{i+1}+x_{i-1}-2 x_{i}\right)+\alpha\left(\left(x_{i+1}^{2}-x_{i}^{2}\right)-\left(x_{i}-x_{i-1}\right)^{2}\right), 1 \leq i \leq N
$$

\footnotetext{
${ }^{3}$ See description by Göktas and Hereman [24] and references therein.
} 

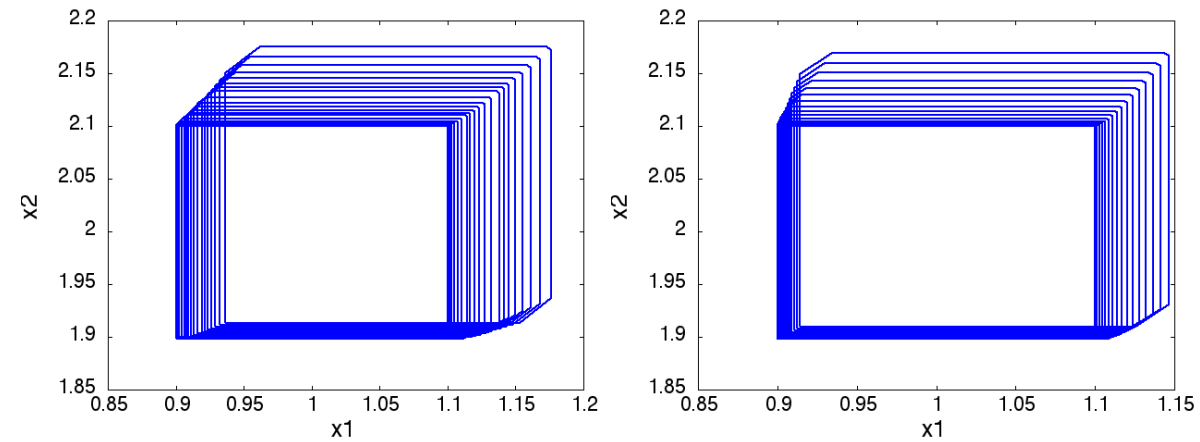

Figure 6: Comparing the flowpipes constructed for Flow* for the Fermi et al. system. (Left) The original 7 state model and (Right) The augmented model with 8 state variables.

We consider an instantiation with $N=3$, searching for CoB transformations with an initial basis of monomials of degree upto 4 . We obtain a transformation representing a conserved quantity

$$
\begin{gathered}
\frac{1}{2}\left(v_{1}^{2}+v_{2}^{2}+v_{3}^{2}\right)+x_{1}^{2}+x_{2}^{2}+x_{3}^{2}-3 x_{3}\left(1+3 a-a x_{3}\right) \\
-x_{2} x_{3}\left(1+a x_{3}-a x_{2}\right)-x_{1} x_{2}\left(1+a x_{2}-a x_{1}\right)
\end{gathered}
$$

The abstract system is given by $\frac{d w_{1}}{d t}=0$.

Figure 6 shows the Flow* comparison of the original system and the augmented one. Whereas the Flow* terminated in 18 seconds for the original system, it required 1292.5 seconds for the augmented system. In our experience, this extra time is not entirely due to the single additional variable but chiefly includes the time taken for the domain contraction operation that is required for enforcing mode invariants [9]. The Flowpipe for the augmented system is, in fact, somewhat more accurate especially comparing the upper bounds for $x_{1}$ established by both Flowpipes.

Two Mass Spring System: Consider the dynamics of two masses connected by a spring to each other and to two fixed walls. The state variables are $\left(x_{1}, x_{2}, v_{1}, v_{2}\right)$ indicating the position and velocity of the masses while the spring constant $k$ is a parameter. The dynamics are given by

$$
\begin{aligned}
\frac{d x_{1}}{d t} & =v_{1} & \frac{d x_{2}}{d t} & =v_{2} \\
\frac{d v_{1}}{d t} & =k x_{2}-2 k x_{1} & \frac{d v_{2}}{d t} & =k\left(x_{1}-x_{2}\right)
\end{aligned}
$$

Our procedure yields a change-of-bases transformation

$w_{1}: v_{2}^{2}+v_{1}^{2}+k x_{2}^{2}-2 k x_{1} x_{2}+2 k x_{1}^{2}, w_{2}: v_{1} v_{2}-\frac{1}{2} v_{1}^{2}-\frac{1}{2} k x_{2}^{2}+2 k x_{1} x_{2}-\frac{3}{2} k x_{1}^{2}$

Both $w_{1}, w_{2}$ represent conserved quantities, yielding the abstraction

$$
\frac{d w_{1}}{d t}=\frac{d w_{2}}{d t}=0 \text {. }
$$



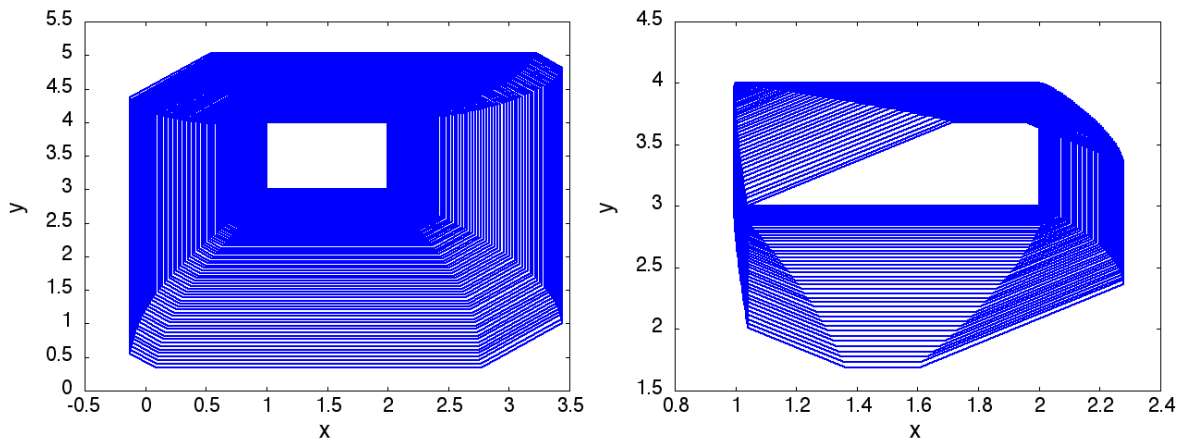

Figure 7: Comparing the flowpipes constructed for Flow* for the two mass spring system. (Left) The original 5 state model and (Right) The augmented model with 7 state variables.

We applied Flow* to this system with initial states

$$
x_{1} \in[1,2], x_{2} \in[3,4], v_{1} \in[0,0.1], v_{2} \in[-0.1,0], k \in[0.5,1.2] .
$$

Flow* requires 8.8 seconds for the original model and 660.5 seconds to analyze the augmented model. The comparison is shown in Figure 7. The flowpipe constructed for the augmented model is clearly more accurate.

Biochemical reaction network: We consider a biochemical reaction network benchmark from Dang et al. [17]. The ODE along with the values are parameters in our model coincide with those used by Dang et al. The ODE consists of 12 variables and roughly 14 parameters. Our search for degree bound $\leq 3$ discovers a transformation generated by five basis functions (in roughly 3 seconds). The model itself is too large to be directly analyzed by Flow*.

Collision Avoidance We consider the algebraic abstraction of the roundabout mode of a collision avoidance system analyzed recently by Platzer et al. [46] and earlier by Tomlin et al. [60]. The two airplane collision avoidance system consists of the variables $\left(x_{1}, x_{2}\right)$ denoting the position of the first aircraft, $\left(y_{1}, y_{2}\right)$ for the second aircraft, $\left(d_{1}, d_{2}\right)$ representing the velocity vector for aircraft 1 and $\left(e_{1}, e_{2}\right)$ for aircraft 2. $\omega, \theta$ abstract the trigonometric terms. In addition, the parameters $a, b, r_{1}, r_{2}$ are also represented as system variables. The dynamics are modeled by the following differential equations:

$$
\begin{array}{ccccc}
x_{1}^{\prime}=d_{1} & x_{2}^{\prime}=d_{2} & d_{1}^{\prime}=-\omega d_{2} & d_{2}^{\prime}=\omega d_{1} \\
y_{1}^{\prime}=e_{1} & y_{2}^{\prime}=e_{2} & e_{1}^{\prime}=-\theta e_{2} & e_{2}^{\prime}=\theta e_{1} \\
a^{\prime}=0 & b^{\prime}=0 & r_{1}^{\prime}=0 & r_{2}^{\prime}=0
\end{array}
$$

A search for transformations of degree 2 yields a closed vector space with 27 basis functions within 0.2 seconds. The basis functions include $a, b, r_{1}, r_{2}$ and all degree two terms involving these. Removing these from the basis, gives us 14 basis functions that yield a transformation to a 14 dimensional affine ODE. 
Table 1: Experimental evaluation results on non linear polynomial ODE benchmarks at a glance. Legend: $|\mathbf{V}|$ denotes number of system variables + parameters, Deg.: max. degree of the system RHS, $\left|\mathbf{D}_{\mathbf{0}}\right|$ : degree limit for monomials in the initial basis, Time: timing in seconds, $\left|\mathbf{B}^{*}\right|$ : number of polynomials in the final basis, $\dagger$ : some elements of the basis involving just the parameters were discarded from the count and dnf: did not finish in $2 \mathrm{hrs}$ or out of memory crash. S?: Did the process succeed in discovering an affine ODE abstraction? X indicates failure, whereas $\checkmark$ indicates success.

\begin{tabular}{||l|l|l||l|l|l||l|l|l||l||}
\hline ID & $|V|$ & Deg. & $\left|D_{0}\right|$ & Time & $\left|B^{*}\right|$ & $\left|D_{0}\right|$ & Time & $\left|B^{*}\right|$ & S? \\
\hline Brusselator & 2 & 3 & 3 & 0.01 & 0 & 25 & 2.8 & 0 & X \\
Fitz-Nag. & 2 & 3 & 3 & 0.01 & 0 & 25 & 2.6 & 0 & X \\
Vandepol & 2 & 3 & 3 & 0.01 & 0 & 25 & 1.9 & 0 & X \\
Proj-drag & 4 & 2 & 3 & 0.02 & 8 & 10 & 9.7 & 64 & $\checkmark$ \\
Circular & 4 & 2 & 3 & 6 & 0.01 & 10 & 10.6 & 83 & $\checkmark$ \\
Hamiltonian & $5+1$ & 2 & 3 & 0.02 & $5 \dagger$ & 5 & 1.3 & $20 \dagger$ & $\checkmark$ \\
Two-spring & $4+1$ & 2 & 3 & 0.03 & $2 \dagger$ & 5 & 0.5 & $6 \dagger$ & $\checkmark$ \\
Toda-2 & 7 & 2 & 3 & 0.1 & 22 & 5 & 4.6 & 82 & $\checkmark$ \\
Toda-3 & 10 & 2 & 3 & 0.5 & 38 & 5 & 95 & 169 & $\checkmark$ \\
Toda-5 & 16 & 2 & 3 & 6 & 90 & 5 & 6373 & 559 & $\checkmark$ \\
Toda-10 & 31 & 2 & 3 & 301.5 & 375 & 5 & & dnf & $\checkmark$ \\
FPUT-3 & $6+1$ & 3 & 3 & 0.05 & $0 \dagger$ & 5 & 3.7 & $2 \dagger$ & $\checkmark$ \\
FPUT-5 & $10+1$ & 3 & 3 & 0.4 & 0 & 5 & 231 & 2 & $\checkmark$ \\
Bio-network & 13 & 2 & 3 & 0.07 & 5 & 5 & 4800 & 20 & $\checkmark$ \\
Roundabout & $10+4$ & 2 & 3 & 1.5 & $68 \dagger$ & 5 & 890 & $\geq 600 \dagger$ & $\checkmark$ \\
\hline
\end{tabular}

\subsection{Discrete Systems}

We now describe experimental results on some discrete programs. We used a set of benchmark programs that require non-linear invariants to prove correctness compiled by Enric Carbonell ${ }^{4}$. Our evaluation focuses on a subset of benchmarks that have non-linear assignments or guards in them. The methods presented here converge in a single step with the initial basis whenever the program being considered already has affine updates.

Fermat Factorization: Figure 8 shows a program for finding a factor of a number $N$ near its square root taken from a book by Bressoud [8]. Our analysis initialized with monomials of degree upto 2 over the program variables yields a final basis consisting of 17 polynomials. The resulting affine system is analyzed by a polyhedral analyzer using abstract interpretation to yield invariants. The invariants obtained at the loop head are shown in Figure 8. The equality invariant

$$
4 r+v^{2}-2 v-u^{2}+2 u+4 N=0
$$

\footnotetext{
${ }^{4}$ The benchmark instances are available on-line at http://www.lsi.upc.edu/ erodri/ webpage/polynomial_invariants/list.html.
} 
Table 2: Timings for computing abstractions of discrete systems and analyzing the resulting abstractions. Legend: $|\mathbf{V}|$ denotes number of system variables, Trs: number of transitions, Deg.: max. degree of the RHS, $\left|\mathbf{D}_{\mathbf{0}}\right|$ degree limit for monomials in the initial basis, Time: timing in seconds, $\left|\mathbf{B}^{*}\right|$ : number of elements in the final basis, \#I: invariants computed and DNF: did not finish in 2 hrs or out of memory crash.

\begin{tabular}{||l|l|l|l||l|l|l||l|l||}
\hline \multicolumn{4}{||c||}{ System } & \multicolumn{3}{c||}{ Linearization } & \multicolumn{2}{c||}{ Analysis } \\
\hline ID & \#V & \#Trs & Deg & $\left|D_{0}\right|$ & $\left|\mathbf{B}^{*}\right|$ & Time & Time & \#I \\
\hline \hline Petter2 & 2 & 1 & 2 & 2 & 3 & 0.02 & $\leq 0.01$ & 10 \\
Petter3 & 2 & 1 & 2 & 3 & 1 & 0.02 & $\leq 0.01$ & 2 \\
Petter3 & 2 & 1 & 3 & 3 & 4 & 0.02 & $\leq 0.01$ & 25 \\
Geo & 6 & 2 & 2 & 2 & 6 & 0.02 & $\leq 0.01$ & 9 \\
Fermat & 5 & 6 & 2 & 2 & 17 & 0.04 & 0.5 & 26 \\
Prodbr & 7 & 5 & 2 & 2 & 20 & 0.06 & 2.0 & 19 \\
Euclidex1 & 11 & 5 & 2 & 2 & 51 & 0.66 & \multicolumn{2}{|c||}{ DNF } \\
\hline
\end{tabular}

[2] Rajeev Alur, Thomas A. Henzinger, G. Lafferriere, and George Pappas. Discrete abstractions of hybrid systems. Proc. of IEEE, 88(7):971-984, 2000 .

[3] Eugene Asarin, Thao Dang, and Oded Maler. The d/dt tool for verification of hybrid systems. In $C A V$, volume 2404 of $L N C S$, pages $365-370$. springer, 2002.

[4] Roberto Bagnara, Enric Rodríguez-Carbonell, and Enea Zaffanella. Generation of basic semi-algebraic invariants using convex polyhedra. In 12th International Symposium on Static Analysis (SAS'05), volume 3672 of Lecture Notes in Computer Science, pages 19-34. Springer-Verlag, September 2005.

[5] Spring Berman, Adam Halasz, and Vijay Kumar. MARCO: a reachability algorithm for multi-affine systems with applications to biological systems. In Hybrid Systems: Computation and Control, volume 4416, pages 76-89. Springer-Verlag, 2007.

[6] M. Berz. Modern Map Methods in Particle Beam Physics, volume 108 of Advances in Imaging and Electron Physics. Academic Press, 1999.

[7] M. Berz and K. Makino. Verified integration of ODEs and flows using differential algebraic methods on high-order Taylor models. Reliable Computing, 4:361-369, 1998.

[8] David M. Bressoud. Factoring and Primality Testing. Springer-Verlag (Undergraduate Texts in Mathematics), 1989.

[9] Xin Chen, Erika Ábraham, and Sriram Sankaranarayanan. Taylor model flowpipe construction for non-linear hybrid systems. In Real Time Systems Symposium (RTSS). IEEE Press, 2012. 
[10] Xin Chen, Erika Ábrahám, and Sriram Sankaranarayanan. Flow*: An analyzer for non-linear hybrid systems. In Computer Aided Verification, volume 8044 of Lecture Notes in Computer Science, pages 258-263. Springer, 2013.

[11] Edmund M. Clarke, Orna Grumberg, Somesh Jha, Yuan Lu, and Helmut Veith. Counterexample-guided abstraction refinement for symbolic model checking. J. ACM, 50(5):752-794, 2003.

[12] G.E. Collins. Quantifier elimination for real closed fields by cylindrical algebraic decomposition. In H.Brakhage, editor, Automata Theory and Formal Languages, volume 33 of LNCS, pages 134-183. Springer, 1975.

[13] George E. Collins and Hoon Hong. Partial cylindrical algebraic decomposition for quantifier elimination. Journal of Symbolic Computation, 12(3):299-328, sep 1991.

[14] Michael Colón. Approximating the algebraic relational semantics of imperative programs. In $11^{\text {th }}$ Static Analysis Symposium (SAS'2004), volume 3148 of LNCS. Springer, 2004.

[15] Patrick Cousot and Nicholas Halbwachs. Automatic discovery of linear restraints among the variables of a program. In $P O P L^{\prime} ' 78$, pages 84-97, January 1978.

[16] David Cox, John Little, and Donald O'Shea. Ideals, Varieties and Algorithms: An Introduction to Computational Algebraic Geometry and Commutative Algebra. Springer-Verlag, 1991.

[17] Thao Dang, Oded Maler, and Romain Testylier. Accurate hybridization of nonlinear systems. In $H S C C$ '10, pages 11-20. ACM, 2010.

[18] Andreas Dolzmann and Thomas Sturm. REDLOG: Computer algebra meets computer logic. ACM SIGSAM Bulletin, 31(2):2-9, June 1997.

[19] Lorenzo Farina and Sergio Rinaldi. Positive Linear Systems: Theory and Applications. Wiley interscience, New York, 2000.

[20] Enrico Fermi, John Pasta, and Stanislaw Ulam. Studies of non-linear problems. Document LA-140, Los Alamos National Laboratories, 1955.

[21] Goran Frehse. PHAVer: Algorithmic verification of hybrid systems past HyTech. In $H S C C$, volume 2289 of $L N C S$, pages 258-273. Springer, 2005.

[22] Goran Frehse, Colas Le Guernic, Alexandre Donzé, Scott Cotton, Rajarshi Ray, Olivier Lebeltel, Rodolfo Ripado, Antoine Girard, Thao Dang, and Oded Maler. Spaceex: Scalable verification of hybrid systems. In Proc. $C A V$, LNCS. Springer, 2011.

[23] Antoine Girard. Reachability of uncertain linear systems using zonotopes. In $H S C C$, volume 3414 of $L N C S$, pages 291-305. Springer, 2005. 
[24] Ünal Göktas and Willy A. Hereman. Symbolic computation of conservation laws, generalized symmetries, and recursion operators for nonlinear differential-difference equations. In Dynamical Systems and Methods. Springer-Verlag, 2011.

[25] Colas Le Guernic and Antoine Girard. Reachability analysis of linear systems using support functions. Nonlinear Analysis: Hybrid Systems, $4(2): 250-262,2010$.

[26] Nicolas Halbwachs, Yann-Eric Proy, and Patrick Roumanoff. Verification of real-time systems using linear relation analysis. Formal Methods in System Design, 11(2):157-185, 1997.

[27] Paul Halmos. Finite-Dimensional Vector Spaces. Springer-Verlag, 1974.

[28] M. Karr. Affine relationships among variables of a program. Acta Informatica, 6:133-151, 1976.

[29] Laura Kovács. Reasoning algebraically about p-solvable loops. In Tools and Algorithms for the Construction and Analysis of Systems (TACAS), volume 4963 of Lecture Notes in Computer Science, pages 249-264. Springer, 2008.

[30] K. Kowalski and W-H. Steeb. Non-Linear Dynamical Systems and Carleman Linearization. World Scientific, 1991.

[31] Alexander B. Kurzhanski and Pravin Varaiya. Ellipsoidal techniques for reachability analysis. In $H S C C$, volume 1790 of $L N C S$, pages 202-214. Springer, 2000.

[32] John M. Lee. Introduction to Smooth Manifolds. Graduate Texts in Mathematics. Springer-Verlag, 2003.

[33] Zohar Manna and Amir Pnueli. Temporal Verification of Reactive Systems: Safety. Springer, New York, 1995.

[34] Nadir Matringe, Arnoldo Viera Moura, and Rachid Rebiha. Morphisms for non-trivial non-linear invariant generation for algebraic hybrid systems. In HSCC, volume 5469 of LNCS, pages 445-449, 2009.

[35] James D. Meiss. Differential Dynamical Systems. SIAM publishers, 2007.

[36] Steven S. Muchnick. Advanced Compiler Design and Implementation. Morgan Kaufmann Publishers Inc., 1997.

[37] Markus Müller-Olm and Helmut Seidl. A note on Karr's algorithm. In Proc. Intl. Conference on Automata, Languages and Programming (ICALP), volume 3142 of $L N C S$, pages 1016-1028, 2004.

[38] Markus Müller-Olm and Helmut Seidl. Precise interprocedural analysis through linear algebra. In Principles Of Programming Languages (POPL), pages 330-341. ACM, 2004. 
[39] K. R. Jackson Ned S. Nedialkov and G. F. Corliss. Validated solutions of initial value problems for ordinary differential equations. Applied Mathematics and Computation, 105(1):21-68, 1999.

[40] Meeko Oishi, Ian Mitchell, Alexandre M. Bayen, and Claire J. Tomlin. Invariance-preserving abstractions of hybrid systems: Application to user interface design. IEEE Trans. on Control Systems Technology, 16(2), Mar 2008.

[41] Pablo A Parillo. Semidefinite programming relaxation for semialgebraic problems. Mathematical Programming Ser. B, 96(2):293-320, 2003.

[42] Marko Petkovsek, Herbert Wilf, and Doron Zeilberger. $A=B$. A K Peters/CRC Press, 1996.

[43] Michael Petter. Berechnung von polynomiellen invarianten (German), October 2004. Cf. http://www2.cs.tum.edu/ petter/da/da.pdf .

[44] André Platzer. Differential-algebraic dynamic logic for differential-algebraic programs. J. Log. Comput., 20(1):309-352, 2010.

[45] André Platzer. The structure of differential invariants and differential cut elimination. Logical Methods in Computer Science, 8(4):1-38, 2012.

[46] André Platzer and Edmund Clarke. Computing differential invariants of hybrid systems as fixedpoints. Formal Methods in Systems Design, 35(1):98120, 2009.

[47] Pavithra Prabhakar, Geir E. Dullerud, and Mahesh Viswanathan. Preorders for reasoning about stability. In $H S C C$, pages 197-206, 2012.

[48] Nacim Ramdani, Nacim Meslem, and Yves Candau. Reachability of uncertain nonlinear systems using a nonlinear hybridization. In $H S C C$, volume 4981 of $L N C S$, pages 415-428. Springer, 2008.

[49] Enric Rodríguez-Carbonell and Deepak Kapur. An Abstract Interpretation Approach for Automatic Generation of Polynomial Invariants. In International Symposium on Static Analysis (SAS 2004), volume 3148 of Lecture Notes in Computer Science, pages 280-295. Springer-Verlag, 2004.

[50] Enric Rodríguez-Carbonell and Deepak Kapur. Automatic Generation of Polynomial Loop Invariants: Algebraic Foundations. In International Symposium on Symbolic and Algebraic Computation 2004 (ISSAC04), pages 266-273. ACM Press, 2004.

[51] Sriram Sankaranarayanan. Automatic invariant generation for hybrid systems using ideal fixed points. In Hybrid Systems: Computation and Control, pages 211-230. ACM Press, 2010. 
[52] Sriram Sankaranarayanan. Automatic abstraction of non-linear systems using change of bases transformations. In Hybrid Systems: Computation and Control (HSCC), pages 143-152. ACM, 2011.

[53] Sriram Sankaranarayanan, Michael A. Colón, Henny Sipma, and Zohar Manna. Efficient strongly relational polyhedral analysis. In VMCAI'06, volume 3855/2006 of LNCS, pages 111-125, 2006.

[54] Sriram Sankaranarayanan, Henny Sipma, and Zohar Manna. Non-linear loop invariant generation using Gröbner bases. In ACM Principles of Programming Languages (POPL), pages 318-330. ACM Press, 2004.

[55] Sriram Sankaranarayanan, Henny Sipma, and Zohar Manna. Constructing invariants for hybrid systems. Formal Methods in System Design, 32(1):25$55,2008$.

[56] Sriram Sankaranarayanan, Henny B. Sipma, and Zohar Manna. Fixed point iteration for computing the time-elapse operator. In $H S C C$, LNCS. Springer, 2006.

[57] Mohamed Amin Ben Sassi and Antoine Girard. Computation of polytopic invariants for polynomial dynamical systems using linear programming. Automatica, 48(12):3114 - 3121, 2012.

[58] Bruno I. Silva, K. Richeson, Bruce H. Krogh, and Alongkrit Chutinan. Modeling and verification of hybrid dynamical system using checkmate. In ADPM 2000, 2000. available online from http://www.ece.cmu.edu/ $\sim$ webk/checkmate.

[59] Ashish Tiwari. Abstractions for hybrid systems. Formal Methods in Systems Design, 32:57-83, 2008.

[60] Claire J. Tomlin, George J. Pappas, and Shankar Sastry. Conflict resolution for air traffic management: A study in multi-agent hybrid systems. IEEE Trans. on Aut. Control, 43(4):509-521, April 1998.

[61] S. Vassilyev and S. Ul'yanov. Preservation of stability of dynamical systems under homomorphisms. Differential Equations, 45:1709-1720, 2009.

[62] Feng Zhao. Automatic Analysis and Synthesis of Controllers for Dynamical Systems Based on Phase-Space Knowledge. PhD thesis, 1998. 